\title{
Curvature bound from gravitational catalysis
}

\author{
Holger Gies ${ }^{1,2, *}$ and Riccardo Martini ${ }^{1, \dagger}$ \\ ${ }^{1}$ Theoretisch-Physikalisches Institut, Abbe Center of Photonics, Friedrich Schiller University Jena, \\ Max Wien Platz 1, 07743 Jena, Germany \\ ${ }^{2}$ Helmholtz-Institut Jena, Fröbelstieg 3, D-07743 Jena, Germany
}

(Received 20 February 2018; published 24 April 2018)

\begin{abstract}
We determine bounds on the curvature of local patches of spacetime from the requirement of intact longrange chiral symmetry. The bounds arise from a scale-dependent analysis of gravitational catalysis and its influence on the effective potential for the chiral order parameter, as induced by fermionic fluctuations on a curved spacetime with local hyperbolic properties. The bound is expressed in terms of the local curvature scalar measured in units of a gauge-invariant coarse-graining scale. We argue that any effective field theory of quantum gravity obeying this curvature bound is safe from chiral symmetry breaking through gravitational catalysis and thus compatible with the simultaneous existence of chiral fermions in the low-energy spectrum. With increasing number of dimensions, the curvature bound in terms of the hyperbolic scale parameter becomes stronger. Applying the curvature bound to the asymptotic safety scenario for quantum gravity in four spacetime dimensions translates into bounds on the matter content of particle physics models.
\end{abstract}

DOI: 10.1103/PhysRevD.97.085017

\section{INTRODUCTION}

Gravitational catalysis denotes the breaking of chiral symmetry and subsequent fermionic mass generation induced by a curved spacetime background. The phenomenon is known to occur generically in fermionic systems of any dimension for various negatively curved spacetimes even at the weakest fermionic attraction [1-17].

Gravitational catalysis can be understood as a consequence of dimensional reduction of the fluctuation spectrum. For instance in $D$-dimensional hyperbolic space, the low-lying modes of the Dirac operator exhibit a reduction from $D$ to $1+1$ dimensions [18]. Hence, the long-range dynamics of any self-interaction of the fermions (be it fundamental, effective or induced) involving a chiral symmetry-breaking channel behaves like the corresponding model in $1+1$ dimensions, e.g., the Gross-Neveu or the Nambu-Jona-Lasinio model, which both exhibit chiral symmetry breaking and fermionic mass generation.

In this respect, gravitational catalysis is closely related to magnetic catalysis [19-24] of chiral symmetry breaking in a magnetic field where a dimensional reduction mechanism is also visible in the fermionic fluctuation spectrum in the form

\footnotetext{
*holger.gies@uni-jena.de

riccardo.martini@uni-jena.de
}

Published by the American Physical Society under the terms of the Creative Commons Attribution 4.0 International license. Further distribution of this work must maintain attribution to the author(s) and the published article's title, journal citation, and DOI. Funded by SCOAP. of the lowest Landau level. Consequently, also the combination of gravitational and gauge-field catalysis exhibits a rich interplay [25-30]. Both phenomena can also be understood within a renormalization group (RG) framework $[31,32]$, where an analysis of the RG flow reveals that the chiral channels inevitably become relevant operators even in higher dimensions, once the long-range flow is driven by the low-lying modes of the fermion spectrum.

Unlike the case of magnetic catalysis, the relevance of gravitational catalysis for real systems is less clear. While the mechanism still works in negatively curved space (instead of spacetime) such as on the Lobachevsky plane [14,32], an estimate for the required curvature for inducing a chiral transition in layered materials with Dirac fermionic excitations, i.e., a Mott transition, results in large negative values which seem difficult to achieve with current materials [32].

In the present work, we argue that gravitational catalysis may play a malign role for the interplay of quantum gravitational and fermionic matter degrees of freedom in the high-energy regime near the Planck scale. As suggested in [33], the observational fact of the existence of light chiral fermions in our Universe puts implicit bounds on the properties of the quantum gravitational interactions: if quantum gravity near the Planck scale was such that it triggered chiral symmetry breaking, the low-energy particle sector of our Universe would generically be characterized by massive fermions with Planck scale masses. As gravity couples equally to all matter degrees of freedom, it thus would seem difficult to understand the existence of light chiral fermions. 
Despite the fact that gravity represents an attractive interaction among particles, gravitational fluctuations in a quantum-field theory setting surprisingly do not trigger chiral symmetry breaking [33,34]. In this respect, gravity differs substantially from gauge theories or Yukawa interactions. Therefore, the existence of light fermions appears compatible even with a high-energy regime of strongly coupled gravity, as long as an effective field theory description for quantum gravity is suitable. In particular, the asymptotic safety scenario of quantum gravity [35-41] passes this consistency test [42-52], also in conjunction with further gauge interactions [53-56]. In asymptotic safety, it is even possible to study the interplay of nonchiral fermions and gravity [57], demonstrating that chiral fermions are favored by simple asymptotic safety scenarios. Certain asymptotically safe gravity-matter scenarios even exhibit an enhanced predictive power [58-65].

Whereas most of these studies have essentially been performed on flat space, with curvature-dependent calculations coming up only recently [66,67], the gravitational catalysis mechanism is active on negatively curved spacetimes. In this picture, the consistency of quantum gravity and light fermions thus is not so much a matter of gravitational fluctuations and their interplay with matter, but of the effective spacetime resulting from quantum gravity itself.

In order to elucidate the mechanism by which gravitational catalysis can affect the realization of quantum gravity and its interplay with the particle content of Nature, we perform a scale-dependent analysis of gravitational catalysis. We introduce an infrared (IR) scale $k_{\mathrm{IR}}$ that serves as a coarse-graining scale for the fermionic long-range modes that drive chiral symmetry breaking. Simultaneously, this scale can be viewed as an inverse length scale of a local patch of spacetime characterized by an averaged curvature. The relevance or irrelevance of gravitational catalysis then arises as a competition between the local curvature-induced contributions and the screening of contributions from the long-range modes. This results in bounds on the local curvature measured in units of the coarse-graining scale: in order to evade fermion mass generation and chiral symmetry breaking, the curvature bound has to be satisfied on all scales $k_{\mathrm{IR}}$.

This statement may become particularly relevant for a high-energy scale of quantum gravity, where $k_{\mathrm{IR}}$ may be of the order of the Planck scale. If a quantum gravity scenario violates our curvature bound in the Planck regime, the possible onset of gravitational catalysis can give rise to a fermion mass spectrum of the corresponding particle physics sector which is expected to be of Planck scale as well. Hence, a violation of the bound can be indicative for a tension between a quantum gravity scenario and the existence and observation of light chiral fermions in our Universe.

Our paper is organized as follows: Sec. II lays out the general framework of our study in terms of a generic chiral fermion theory in curved spacetime, which we analyze in a local mean-field RG approach. We illustrate our approach with the fully analytically accessible simplest case of $D=3$ dimensional spacetime. The most relevant $D=4$ dimensional case is analyzed in Sec. III. Higherdimensional cases are studied in Sec. IV, where we find that the curvature bound gets stronger with increasing dimensionality. We illustrate the usefulness of the curvature bound with the aid of the asymptotic safety scenario for quantum gravity in a simple setting in Sec. V. In this scenario, the curvature bound can, for instance, translate into a bound on the admissible number of fermion flavors. We conclude in Sec. VI.

\section{FRAMEWORK}

Let us start from a fermionic matter sector with a global chiral symmetry group $U\left(N_{\mathrm{f}}\right)_{\mathrm{R}} \times U\left(N_{\mathrm{f}}\right)_{\mathrm{L}}$, with $N_{\mathrm{f}}$ being the number of fermion species. This is reminiscent to the fermionic sector of the standard model subject to the strong interaction with $N_{\mathrm{f}}$ counting the number of flavors times the number of colors. Even without any further gauge interactions, gravitational fluctuations, say in the (trans-) Planckian regime, will induce effective fermionic selfinteractions. With gravity preserving chiral symmetry, the most general local fermionic self-interaction to fourth-order in the fields is parametrized by the action $[33,68]$

$$
\begin{aligned}
& S[\bar{\psi}, \psi]=\int_{x}\{\bar{\psi} \not \phi \psi \\
& +\frac{\bar{\lambda}_{-}}{2}\left[\left(\bar{\psi}^{a} \gamma_{\mu} \psi^{a}\right)^{2}+\left(\bar{\psi}^{a} \gamma_{\mu} \gamma_{5} \psi^{a}\right)^{2}\right] \\
& \left.+\frac{\bar{\lambda}_{+}}{2}\left[\left(\bar{\psi}^{a} \gamma_{\mu} \psi^{a}\right)^{2}-\left(\bar{\psi}^{a} \gamma_{\mu} \gamma_{5} \psi^{a}\right)^{2}\right]\right\},
\end{aligned}
$$

where the Latin indices represent different flavor species and $\mathbb{Z}$ is the covariant Dirac operator. Denoting the vector interaction channel term with $(V)=\left(\bar{\psi} \gamma_{\mu} \psi\right)^{2}$ and the axial one with $(A)=-\left(\bar{\psi} \gamma_{\mu} \gamma_{5} \psi\right)^{2}$, we expect the transition to be triggered by the $(V)+(A)$ term which is equivalent to

$$
(V)+(A)=-2\left[\left(S^{\mathrm{N}}\right)-\left(P^{\mathrm{N}}\right)\right]
$$

by means of a Fierz transformation. Here, $\left(S^{\mathrm{N}}\right)$ and $\left(P^{\mathrm{N}}\right)$ denote the scalar and pseudo scalar channels in the space of flavor nonsinglet terms,

$$
\begin{aligned}
& \left(S^{\mathrm{N}}\right)=\left(\bar{\psi}^{a} \psi^{b}\right)^{2}=\left(\bar{\psi}^{a} \psi^{b}\right)\left(\bar{\psi}^{b} \psi^{a}\right), \\
& \left(P^{\mathrm{N}}\right)=\left(\bar{\psi}^{a} \gamma_{5} \psi^{b}\right)^{2}=\left(\bar{\psi}^{a} \gamma_{5} \psi^{b}\right)\left(\bar{\psi}^{b} \gamma_{5} \psi^{a}\right) .
\end{aligned}
$$

In fact, the structure $\left(S^{\mathrm{N}}\right)-\left(P^{\mathrm{N}}\right)$ is familiar from the Nambu-Jona-Lasinio (NJL) model and further generic models of chiral symmetry breaking. In such models, the 
onset of chiral symmetry breaking is signaled by this channel becoming RG relevant. For instance, in the NJL model, this onset is triggered by a choice of the fourfermion coupling being larger than some critical value. Hence, we concentrate in the following on the NJL channel and ignore the $(V)-(A)$ channel for the rest of the paper. The latter is expected to stay RG irrelevant across a possible phase transition, justifying to approximate $\lambda_{-} \simeq 0$ for the purpose of detecting the onset of symmetry breaking.

Using the projectors on the left and right chiral components

$P_{\mathrm{L}}=\frac{\mathbb{1}-\gamma_{5}}{2}, \quad P_{\mathrm{R}}=\frac{\mathbb{1}+\gamma_{5}}{2}, \quad \mathbb{1}=P_{\mathrm{L}}+P_{\mathrm{R}}$,

the NJL channel can also be written as the interaction part of the Lagrangian

$$
\mathcal{L}_{\text {int }}(\bar{\psi}, \psi)=-2 \bar{\lambda}\left(\bar{\psi}_{\mathrm{L}}^{a} \psi_{\mathrm{R}}^{b}\right)\left(\bar{\psi}_{\mathrm{R}}^{b} \psi_{\mathrm{L}}^{a}\right), \quad \bar{\lambda}=2 \bar{\lambda}_{+} .
$$

Here, the subscripts L, R represent the chiral projections of the Dirac fermion. By means of a Hubbard-Stratonovich transformation, the interaction term can also be expressed in terms of a Yukawa interaction with an auxiliary scalar field,

$$
\mathcal{L}_{\text {int }}(\phi, \bar{\psi}, \psi)=\bar{\psi}^{a}\left[P_{\mathrm{L}}\left(\phi^{\dagger}\right)_{a b}+P_{\mathrm{R}} \phi_{a b}\right] \psi^{b}+\frac{1}{2 \bar{\lambda}} \operatorname{tr}\left(\phi^{\dagger} \phi\right) .
$$

The equivalence of Eq. (6) with Eq. (5) becomes obvious with the help of the equations of motion for the chiral matrix fields $\phi$ and $\phi^{\dagger}$,

$$
\begin{aligned}
\phi_{a b} & =-2 \bar{\lambda} \bar{\psi}_{\mathrm{R}}^{b} \psi_{\mathrm{L}}^{a}, \\
\left(\phi^{\dagger}\right)_{a b} & =-2 \bar{\lambda} \bar{\psi}_{\mathrm{L}}^{b} \psi_{\mathrm{R}}^{a} .
\end{aligned}
$$

From Eq. (6), it is obvious that the Dirac particles can acquire a mass if chiral symmetry gets broken by a nonzero expectation value of the field $\phi_{a b}$. The precise breaking pattern is fixed by the nonzero components of $\left\langle\phi_{a b}\right\rangle$ which in turn is determined by the minima of the effective potential for $\phi$. In the following, we assume a diagonal breaking pattern, $\phi_{a b}=\phi_{0} \delta_{a b}$ with constant order parameter $\phi_{0}$, which for $\left|\phi_{0}\right|>0$ breaks the chiral group down to a residual vector symmetry familiar from QCD-like theories. In the form of Eq. (6) read together with the fermion kinetic term, we can integrate out the fermionic degrees of freedom and obtain the standard mean-field expression for the effective potential of the order parameter

$$
\begin{aligned}
U\left(\phi_{0}\right) & =\frac{N_{\mathrm{f}}}{2 \bar{\lambda}} \phi_{0}^{2}-N_{\mathrm{f}} \log \operatorname{det}\left(\not \nabla+\phi_{0}\right) \\
& =\frac{N_{\mathrm{f}}}{2 \bar{\lambda}} \phi_{0}^{2}-\frac{N_{\mathrm{f}}}{2} \operatorname{Tr} \log \left(-\mathbb{Z}^{2}+\phi_{0}^{2}\right),
\end{aligned}
$$

where we have made use of $\gamma_{5}$-Hermiticity of the Dirac operator in the last step. Since we are considering a homogeneous order parameter, the trace (as well as log det) is understood to be already normalized by a spacetime volume factor, such that we are considering local quantities throughout the paper. Using the Schwinger proper time representation, we write

$$
U(\phi)=\frac{N_{\mathrm{f}}}{2 \bar{\lambda}} \phi_{0}^{2}+\frac{N_{\mathrm{f}}}{2} \int_{0}^{\infty} \frac{d T}{T} e^{-\phi_{0}^{2} T} \operatorname{Tr} e^{\not \chi^{2} T},
$$

where we encounter the trace of the heat kernel on the manifold under consideration,

$$
\operatorname{Tr} e^{\not X^{2} T}=\operatorname{Tr} K\left(x, x^{\prime} ; T\right)=: K_{T} .
$$

The heat kernel $K\left(x, x^{\prime} ; T\right)$ satisfies

$$
\frac{\partial}{\partial T} K=\not \not^{2} K, \quad \lim _{T \rightarrow 0^{+}} K\left(x, x^{\prime} ; T\right)=\frac{\delta\left(x-x^{\prime}\right)}{\sqrt{g}} .
$$

In our analysis, the information about the nature of spacetime enters through the trace of the heat kernel of the (squared) Dirac operator. As this trace parametrizes the contributions of fermionic fluctuations on all scales, the explicit evaluation of Eq. (9) would contain information about both the local and global structure of spacetime.

Though the proper time integration has been introduced as an auxiliary representation, the integrand can be interpreted as the result of a diffusion process of a fictitious particle on the spacetime within propagation time $T$ $[69,70]$. The trace enforces that the diffusion path is closed. For a finite proper time $T$, the fictitious particle traces out a closed path in spacetime which is localized around a point $x$ under consideration. This path can be considered as the spacetime path of a virtual fermionic fluctuation; this perspective can also be made explicit by introducing a Feynman path-integral representation of the heat kernel (worldline formalism) [71-75]. For instance, the mean average distance of the diffusing particle from its center of mass in flat space is $d=\sqrt{T / 6}$ [76], indicating that $\sqrt{T}$ can be considered as a typical length scale of the fluctuations at a fixed value of $T$.

Aiming at a statement about spacetime in the (trans-) Planckian regime, we do not want to make an assumption about its global properties, but intend to consider only local patches of spacetime. This is possible by means of an RGtype analysis of Eq. (9). For this, we introduce a proper time regulator function $f_{k}$ inside the proper time integral $[77,78]$,

$$
f_{k}=e^{-\left(k^{2} T\right)^{p}} .
$$

Here, the power $p>0$ is a parameter specifying the details of the regularization and $k$ corresponds to an IR momentum 
space regularization scale. For instance, for $p \rightarrow \infty$, all long-range contributions for length scales $\sqrt{T}>1 / k$ are cut off sharply. For finite $p$, the length scale $1 / k$ becomes a smooth long-range cutoff. The case $p=1$ is special as it corresponds precisely to a Callan-Symanzik regularization scheme. In the limit $k \rightarrow 0$, the insertion factor becomes $f_{k \rightarrow 0}=1$ and the regularization is removed. Starting from the bare potential $U_{\Lambda}$ at a high momentum scale $k=\Lambda$, the potential at any IR scale $k_{\mathrm{IR}}$ can be constructed from

$U_{k_{\mathrm{IR}}}=U_{\Lambda}-\int_{k_{\mathrm{IR}}}^{\Lambda} d k \partial_{k} U_{k}, \quad U_{\Lambda}=\frac{N_{\mathrm{f}}}{2 \bar{\lambda}_{\Lambda}} \phi_{0}^{2}, \quad \bar{\lambda}_{\Lambda} \equiv \bar{\lambda}$,

once the RG flow of the potential is known,

$$
\partial_{k} U_{k}=\frac{N_{\mathrm{f}}}{2} \int_{0}^{\infty} \frac{d T}{T} e^{-\phi_{0}^{2} T} \partial_{k} f_{k} K_{T} .
$$

In Eq. (12), we explicitly appended the subscript $\Lambda$ to the bare coupling $\bar{\lambda}$ in order to highlight that the bare coupling has to be fixed at the high scale in order to define the model.

Since $\partial_{k} f_{k} \sim T^{p}$ for small $T$, also the short-range fluctuations are suppressed in Eq. (13), such that the consequences of the fermionic fluctuations can be studied in a Kadanoff-Wilson-spirit length scale by length scale. The evaluation of one RG step $\sim \partial_{k} U_{k}$, typically receives contributions from length scales $\sqrt{T} \sim 1 / k$. This implies that we do not have to know the global structure of the spacetime, but our assumptions about the spacetime properties need to hold only over these covariant length scales. More specifically, we assume below that the spacetime can locally be approximated as maximally symmetric.

Though the analysis of the chiral interactions leading to Eq. (1) has been performed in $D=4$ dimensional spacetime, the analysis of the flow of the order-parameter potential in Eq. (13) can be performed in any $D$, though the relation to the symmetry-breaking channel can be more involved or not necessarily be unique in other dimensions; see [79] for an analysis in $D=3$. In higher dimensions, the perturbative nonrenormalizability of Yukawa theories suggests that more relevant operators appear near the Gaussian fixed point. The corresponding regularization of UV divergencies may require higher values of $p$ for a stronger suppression of UV modes. Independently of these technical complications, our analysis can in principle be performed in any dimension.

\section{A. $D=3$}

Let us begin with an analysis of the RG flow of the potential for the case of $D=3$ spacetime dimensions. This case is highly instructive from the viewpoint of the method: it can be treated analytically in all detail, and does not involve further relevant operators. Since gravitational catalysis can occur for negative curvature, we consider spacetimes that can locally be approximated by a hyperbolic space for Euclidean signature, corresponding to AdS spacetime for a Lorentzian signature. The analysis could similarly be performed for spacetimes with negative curvature in the purely spatial part with quantitatively rather similar results $[14,32]$. In $D=3$, the trace of the heat kernel reads $[80,81]$

$$
K_{T}=\frac{1}{8 \pi^{\frac{3}{2}} T^{\frac{3}{2}}}\left(1+\frac{1}{2} \kappa^{2} T\right)
$$

where

$$
\kappa^{2}=-\frac{R}{D(D-1)}=-\frac{R}{6} \geq 0,
$$

denotes the local curvature parameter related to the Ricci scalar $R$. Further details of the heat kernels relevant for this work are briefly reviewed in the Appendix.

Including the proper time regularization, this leads to an effective, scale-dependent potential of the following form:

$U_{k}=\frac{N_{\mathrm{f}}}{2 \bar{\lambda}} \phi_{0}^{2}+\frac{N_{\mathrm{f}}}{2(4 \pi)^{\frac{3}{2}}} \int_{0}^{\infty} \frac{d T}{T^{\frac{5}{2}}} e^{-\phi_{0}^{2} T} f_{k}\left(1+\frac{1}{2} \kappa^{2} T\right)$.

In $D=3$, the Callan-Symanzik regulator is known to be sufficient to control the RG flow of our model. Thus, let us first choose the exponent $p=1$ for $f_{k}$ for simplicity; the result for general $p$ will be given below. The regularized flow of the potential with respect to the scale $k$ then reads

$$
\begin{aligned}
\partial_{k} U_{k}(\phi)= & -\frac{2 k N_{\mathrm{f}}}{2(4 \pi)^{\frac{3}{2}}}\left[\int_{0}^{\infty} \frac{d T}{T^{\frac{3}{2}}} e^{-k^{2} T}\left(e^{-\phi_{0}^{2} T}-1\right)\right. \\
& \left.+\frac{\kappa^{2}}{2} \int_{0}^{\infty} \frac{d T}{T^{\frac{1}{2}}} e^{-k^{2} T}\left(e^{-\phi_{0}^{2} T}-1\right)\right] \\
= & \frac{N_{\mathrm{f}}}{4 \pi}\left[k^{2}\left(\sqrt{1+\frac{\phi_{0}^{2}}{k^{2}}}-1\right)-\frac{\kappa^{2}}{4}\left(\frac{k}{\sqrt{\phi_{0}^{2}+k^{2}}}-1\right)\right] .
\end{aligned}
$$

Upon insertion into Eq. (12), the effective potential at the scale $k_{\mathrm{IR}}$ can be computed, yielding

$$
\begin{aligned}
U_{k_{\mathrm{IR}}}= & -\frac{N_{\mathrm{f}}}{2} \phi_{0}^{2}\left(\frac{1}{\bar{\lambda}_{\mathrm{cr}}}-\frac{1}{\bar{\lambda}_{\Lambda}}-\frac{k_{\mathrm{IR}}}{4 \pi}\right) \\
& +\frac{N_{\mathrm{f}}}{12 \pi}\left(\left(\phi_{0}^{2}+k_{\mathrm{IR}}^{2}\right)^{\frac{3}{2}}-\frac{3}{2} k_{\mathrm{IR}} \phi_{0}^{2}-k_{\mathrm{IR}}^{3}\right) \\
& -\frac{N_{\mathrm{f}}}{16 \pi} \kappa^{2}\left(\sqrt{\phi_{0}^{2}+k_{\mathrm{IR}}^{2}}-k_{\mathrm{IR}}\right),
\end{aligned}
$$

where we have introduced the (scheme-dependent) critical coupling $\bar{\lambda}_{\text {cr }}=4 \pi / \Lambda$, and dropped terms of order $\mathcal{O}(1 / \Lambda)$. 
The physics described by this effective potential can be read off line by line: the first line describes the masslike term in the potential. For subcritical coupling $\bar{\lambda}_{\Lambda}<\bar{\lambda}_{\mathrm{cr}}$, the masslike term remains positive for any $k_{\mathrm{IR}}$ implying that the system in flat space remains in the symmetric phase with a minimum $\phi_{0}=0$ and does not develop fermion masses. For supercritical couplings $\bar{\lambda}_{\Lambda}>\bar{\lambda}_{\mathrm{cr}}$, the masslike term becomes negative below a certain critical IR scale $k_{\mathrm{IR}}$, indicating that the potential develops a nontrivial minimum $\phi_{0}^{2}>0$. The system hence exhibits chiral symmetry breaking and fermion mass generation already in flat space. The second line does not contribute to the masslike term $\sim \phi_{0}^{2}$ upon Taylor expansion. For large $\phi_{0}$ it grows $\sim+\phi_{0}^{3}$, ensuring stability of the potential. The third line represents the contribution due to nonzero curvature, being manifestly negative. In the limit $k_{\mathrm{IR}} \rightarrow 0$, it is linear in the field $\phi_{0}$ and thus dominates for small field amplitudes. In this way, it induces a nonzero $\phi_{0}$ and inevitably drives the system to chiral symmetry breaking and fermion mass generation - the essence of gravitational catalysis [1-4, $6-18,26,27,29,30,32]$.

However, gravitational catalysis receives its relevant contributions from the deep IR, i.e., the long-wavelength modes. In order to dominate the mass spectrum, the curvature has to be such that the hyperbolic space is an adequate description also on large length scales. Within our $\mathrm{RG}$ description, we make the less severe assumption that the hyperbolic space is an adequate description only up to lengths scales of order $1 / k_{\mathrm{IR}}$. Whether or not the potential develops a nonzero minimum then is decided by the competition between the first and the third line of Eq. (18).

Since we are interested in curvature-induced symmetry breaking, we assume that the fermionic interactions are subcritical, $\bar{\lambda}_{\Lambda} \leq \bar{\lambda}_{\text {cr }}$, such that the masslike term in the first line is bounded from below by

$$
-\frac{N_{\mathrm{f}}}{2} \phi_{0}^{2}\left(\frac{1}{\bar{\lambda}_{\mathrm{cr}}}-\frac{1}{\bar{\lambda}_{\Lambda}}-\frac{k_{\mathrm{IR}}}{4 \pi}\right) \geq \frac{N_{\mathrm{f}} k_{\mathrm{IR}}}{8 \pi} \phi_{0}^{2} .
$$

The only other term contributing to the masslike term arises from the curvature-dependent third line of Eq. (18):

$$
-\frac{N_{\mathrm{f}}}{16 \pi} \kappa^{2}\left(\sqrt{\phi_{0}^{2}+k_{\mathrm{IR}}^{2}}-k_{\mathrm{IR}}\right)=-\frac{N_{\mathrm{f}} k_{\mathrm{IR}}}{32 \pi} \frac{\kappa^{2}}{k_{\mathrm{IR}}^{2}} \phi_{0}^{2}+\mathcal{O}\left(\phi_{0}^{4}\right) .
$$

Comparing the last two equations tells us that gravitational catalysis does not induce chiral symmetry breaking and fermion mass generation as long as hyperbolic-curvature parameter satisfies

$$
\frac{\kappa^{2}}{k_{\mathrm{IR}}^{2}} \leq 4
$$

In terms of the negative scalar (spacetime) curvature, this implies

$$
|R|=6 \kappa^{2} \leq 24 k_{\mathrm{IR}}^{2} \quad(\text { for } R<0)
$$

inhibits the occurrence of the nontrivial minimum of the effective potential and thus fermion mass generation induced by a negative masslike term. Equation (22) represents our first example of a curvature bound from gravitational catalysis: in line with our assumptions we conclude that a fermionic particle-physics system will not be plagued by curvature-induced chiral symmetry breaking, as long as the local curvature of spacetime patches averaged over the scale of $1 / k_{\mathrm{IR}}$ satisfies the bound (22).

Some comments are in order: (i) from the derivation, it is obvious that a study of the masslike term $\sim \phi_{0}^{2}$ is sufficient to obtain a curvature bound. Of course, the global structure of an effective potential could be such that a nontrivial minimum exists even for a positive masslike term. In that case, the true curvature bound would even be stronger than the one derived from the masslike term. (In the present $D=3$ dimensional system, this does not happen at mean-field level.)

(ii) The curvature bound is independent of the selfcouplings, because of our estimate performed in Eq. (19). The equal sign holds for bare couplings exactly tuned to criticality, i.e., the maximum value of the self-coupling that does not lead to chiral symmetry breaking in the IR. Therefore, the bound limits the curvature and coupling regime where the system is safe from fermion mass generation through gravitational catalysis. Whether or not fermion mass generation sets in if the bound is violated depends on further details of the system such as the fermion couplings.

(iii) The bound is naively scheme-dependent in the sense that the prefactor (24 in the present case) depends on the way the fluctuation averaging procedure is performed. In the calculation so far, we used a Callan-Symanzik regulator that suppresses long-wavelength modes beyond the scale $1 / k_{\mathrm{IR}}$ exponentially. In fact, the calculation can straightforwardly be performed for the general regulator (11). For general $p$, we obtain

$$
\frac{\kappa^{2}}{k_{\mathrm{IR}}^{2}} \leq \frac{2 \Gamma\left(1-\frac{1}{2 p}\right)}{\Gamma\left(1+\frac{1}{2 p}\right)} .
$$

For $p=1$, we obtain (21) and (22) again, whereas we find in the sharp cutoff limit $p \rightarrow \infty$

$$
|R| \leq 12 k_{\mathrm{IR}}^{2}, \quad(\text { for } p \rightarrow \infty, R<0) .
$$

Comparing this to (22), the curvature bound naively seems to be stronger for $p \rightarrow \infty$. However, this simply reflects the fact that the length scale of the fluctuations $1 / k_{\mathrm{IR}}$ is effectively shorter for the sharp cutoff than for the smooth 
exponential regulator, where the fluctuations extending even further out are only suppressed but not cut off. Hence, it is plausible to say that $\left.k_{\mathrm{IR}}\right|_{p \rightarrow \infty}$ is effectively larger than $\left.k_{\mathrm{IR}}\right|_{p=1}$. This goes hand in hand with the inversely behaving prefactor. We consider this as an indication that the curvature bound itself has a schemeindependent meaning: the scheme dependence of the prefactors in the bound should be viewed as a parametrization of the fluctuation averaging process that has to be matched with the procedure that determines the averaged curvature.

\section{III. $D=4$ DIMENSIONAL SPACETIME}

Let us now turn to the physically more relevant case of $D=4$ dimensional spacetime. The analysis is conceptually complicated by the appearance of two more relevant operators coming along with physical couplings, and technically more involved because of the structure of the heat kernel. Nevertheless, it is possible to capture the essential behavior analytically by making use asymptotic heat-kernel expansions and a simple interpolation. The full result is, of course, analyzed below by straightforward numerical integration. We start with the representation of the heat-kernel trace as a one-parameter integral $[80,81]$

$$
K_{T}=\frac{2}{(4 \pi T)^{2}} \int_{0}^{\infty} d u e^{-u^{2}} u\left(u^{2}+\kappa^{2} T\right) \operatorname{coth}\left(\frac{\pi u}{\kappa \sqrt{T}}\right) .
$$

Using the asymptotic expansions of the coth function, cf. Eqs. (A13) and (A14), the weak and strong curvature expansions of the heat kernel read

$$
\begin{gathered}
K_{T}=\frac{2}{(4 \pi T)^{2}}\left(1+\kappa^{2} T+\cdots\right), \quad \kappa^{2} T \ll 1, \\
K_{T}=\frac{1}{(4 \pi T)^{2}}\left(\frac{\kappa^{3} T^{\frac{3}{2}}}{\pi}+\frac{3+\pi^{2}}{6 \sqrt{\pi}} \kappa T^{\frac{1}{2}}+\cdots\right), \quad \kappa^{2} T \gg 1 .
\end{gathered}
$$

For a simple qualitative, still asymptotically exact estimate, we use an interpolating approximation of the heat kernel that allows for a fully analytical treatment,

$$
K_{T} \simeq \frac{2}{(4 \pi T)^{2}}\left(1+\kappa^{2} T+\frac{\kappa^{3} T^{\frac{3}{2}}}{\pi}\right) .
$$

Upon insertion of the heat kernel into Eqs. (12) and (13), a first difference to the $D=3$ case is the occurrence of a logarithmic UV divergence of the type $\sim \phi_{0}^{4} \ln \Lambda$. This is expected as $\phi^{4}$ is a marginal operator in $D=4$, the coupling of which corresponds to a new and independent physical parameter. The proper definition of the particle system requires to also define an initial condition for the flow of this operator, i.e., to put a counterterm at the high scale $\Lambda$. This is then fixed by demanding for a specific physical renormalized value for the $\phi^{4}$ coupling in a longrange experiment.

For our purposes, these details are, in fact, not relevant, as the $\phi^{4}$ coupling cannot inhibit chiral symmetry breaking. Once the masslike term $\sim \phi_{0}^{2}$ triggers the onset of a chiral condensate, the $\phi^{4}$ coupling will take influence on the final value of the condensate $\phi_{0}$; this is, however, irrelevant for the curvature bound. For consistency, we only assume that the renormalized $\phi^{4}$ coupling is such that the potential is stable towards large fields.

As we have seen in the $D=3$ case, we can obtain a curvature bound by solely studying the $\phi_{0}^{2}$ term of the potential. Using the approximate form of the heat kernel (28), we obtain the analytic estimate to this order:

$$
\begin{aligned}
\left.U_{k_{\mathrm{IR}}}\right|_{\phi_{0}^{2}}= & -\frac{N_{\mathrm{f}} \phi_{0}^{2}}{2}\left[\frac{1}{\bar{\lambda}_{\mathrm{cr}}}-\frac{1}{\bar{\lambda}_{\Lambda}}-\Gamma\left(1-\frac{1}{p}\right) \frac{k_{\mathrm{IR}}^{2}}{(4 \pi)^{2}}\right. \\
& \left.+2 \frac{\kappa^{3}}{k_{\mathrm{IR}}} \frac{\Gamma\left(1+\frac{1}{2 p}\right)}{\sqrt{\pi}}\right]-\frac{N_{\mathrm{f}} \phi_{0}^{2}}{(4 \pi)^{2}} \kappa^{2} \log \left(\frac{\Lambda}{k_{\mathrm{IR}}}\right),
\end{aligned}
$$

again dropping terms of order $\mathcal{O}(1 / \Lambda)$. As before, the diverging contribution coming from the flat part of the heat kernel is indicative of the critical value of the coupling constant,

$$
\bar{\lambda}_{\mathrm{cr}}=\frac{(4 \pi)^{2}}{\Lambda^{2} \Gamma\left(1-\frac{1}{p}\right)} .
$$

As a new feature in $D=4$, we observe a new logarithmically divergent term $\sim \ln \Lambda$ in Eq. (29). This term corresponds to a new, power-counting marginal operator of the form $\phi^{2} R$, which again comes along with a new physical parameter to be fixed by renormalization. Hence, we introduce an initial condition for this operator at the high scale with a bare coupling $\xi_{\Lambda}$ :

$$
\left.U_{\Lambda}\right|_{\phi^{2} R}=N_{\mathrm{f}} \xi_{\Lambda} \phi^{2} R
$$

Upon inclusion of Eq. (30), the effective potential at the scale $k_{\mathrm{IR}}$ receives an overall contribution of the form

$$
\begin{aligned}
\left.U_{k_{\mathrm{IR}}}\right|_{\phi^{2} R} & =-\left(\xi_{\Lambda} N_{\mathrm{f}}+\frac{3 N_{\mathrm{f}}}{4 \pi^{2}} \log \left(\frac{\Lambda}{k_{\mathrm{IR}}}\right)\right) \phi_{0}^{2}|R| \\
& \equiv-N_{\mathrm{f}} \xi_{k_{\mathrm{R}}} \phi_{0}^{2}|R|,
\end{aligned}
$$

where we have made use of the relation $\kappa^{2}=\frac{|R|}{12}, R<0$, in $D=4$. Here we have introduced the long-range parameter $\xi_{k_{\mathrm{IR}}}$ that, in principle, has to be fixed by a physical measurement. For our analysis, we will consider it as a free parameter. As a consequence, the curvature bound depends parametrically on this physical coupling. Assuming again that the fermion self-interactions are subcritical $\bar{\lambda}_{\Lambda} \leq \bar{\lambda}_{\mathrm{cr}}$, we obtain again a bound on the curvature parameter for which no chiral symmetry breaking occurs: 


$$
\frac{\kappa^{3}}{k_{\mathrm{IR}}^{3}}+\frac{4}{3} \frac{\pi^{\frac{5}{2}}}{\Gamma\left(1+\frac{1}{2 p}\right)} \xi_{k_{\mathrm{IR}}} \frac{\kappa^{2}}{k_{\mathrm{IR}}^{2}} \leq \frac{\sqrt{\pi}}{2} \frac{\Gamma\left(1-\frac{1}{p}\right)}{\Gamma\left(1+\frac{1}{2 p}\right)} .
$$

The divergence of the right-hand side for $p \rightarrow 1$, where the bound seems to disappear, is an artifact of the CallanSymanzik regulator which is insufficient to control all UV divergences in $D=4$. In order to stay away from this artifact, we consider regulators in the range $p \in[2, \infty]$.

For a comparison with the $D=3$ case, let us first set $\xi_{k_{\mathrm{R}}}=0$ and consider limiting regulator values,

$$
\left.\frac{\kappa^{3}}{k_{\mathrm{IR}}^{3}}\right|_{p=2} \leq \frac{\sqrt{\pi}}{2} \frac{\Gamma\left(\frac{1}{2}\right)}{\Gamma\left(\frac{5}{4}\right)},\left.\quad \frac{\kappa^{3}}{k_{\mathrm{IR}}^{3}}\right|_{p \rightarrow \infty} \leq \frac{\sqrt{\pi}}{2} .
$$

From (32), it is obvious that the bound gets stronger (weaker) for positive (negative) coupling $\xi_{k_{\mathrm{IR}}}$. Most importantly, there is a nontrivial bound for any finite value of $\xi_{k_{\mathbb{R}}}$.

While Eq. (33) has been derived analytically based on the interpolating approximation (28) for the heat kernel, a full calculation can be performed numerically. For this, we first have to isolate the divergent pieces by hand, treat them analytically as before. In fact, all divergent parts are related to the small curvature expansion of the heat kernel, i.e., to the expansion coefficients displayed in Eq. (26). Treating them separately as before leaves us with a triple integral over the heat kernel parameter $u$ in Eq. (25), the propertime $T$ and the RG scale $k$. A transition to dimensionless integration variables $t=\kappa^{2} T$ and $\sigma=k / \kappa$ yields an integral representation depending only on the dimensionless parameter ratio $\kappa / k_{\mathrm{IR}}$. The masslike term of the effective potential then acquires the form

$$
\begin{aligned}
\left.U_{k_{\mathrm{IR}}}\right|_{\phi_{0}^{2}}= & -\frac{N_{\mathrm{f}} \phi_{0}^{2}}{2}\left[\frac{1}{\bar{\lambda}_{\mathrm{cr}}}-\frac{1}{\bar{\lambda}_{\Lambda}}+\kappa^{2} \mathcal{A}\left(\frac{\kappa}{k_{\mathrm{IR}}} ; p\right)\right] \\
& -12 N_{\mathrm{f}} \xi_{k_{\mathrm{IR}}} \phi_{0}^{2} \kappa^{2},
\end{aligned}
$$

with the function $\mathcal{A}$ to be evaluated by numerical integration. Assuming subcritical fermion interactions $\bar{\lambda}_{\Lambda} \leq \bar{\lambda}_{\text {cr }}$, the curvature bound can be expressed as

$$
\frac{1}{2} \mathcal{A}\left(\frac{\kappa}{k_{\mathrm{IR}}} ; p\right)+12 \xi_{\mathrm{IR}_{\mathrm{R}}} \geq 0
$$

in order to avoid fermion mass generation from gravitational catalysis. The function $\mathcal{A}$ is plotted in Fig. 1 as a function of $k_{\mathrm{IR}} / \kappa$ for $p=2$ (solid line). For comparison, the dashed line represents the result from the analytical interpolation matching the full behavior qualitatively for all curvatures. The strong and weak curvature asymptotics matches very well: we have checked that the leading power laws for both results are the same with coefficients agreeing within an error below the $1 \%$ level. In the intermediate curvature region, the deviations between the numerical result and the analytical estimate are larger.

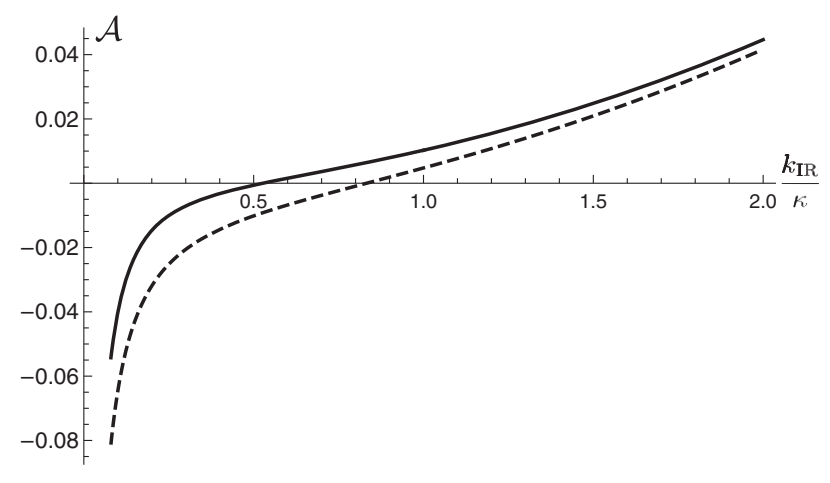

---_- Analytical _ Numerics

FIG. 1. Scaling of the masslike term of the effective potential: the function $\mathcal{A}$, cf. Eq. (34), (solid line) as a function of the inverse curvature parameter $k_{\mathrm{IR}} / \kappa$ is compared to the analytical approximation obtained, cf. Eq. (29), (dashed line) for the case $p=2$. For the case of $\xi_{k_{\mathrm{I}}}=0$, positive values of $\mathcal{A}$ are compatible with the absence of chiral symmetry breaking and the existence of chiral fermions at low energies. The zero crossing corresponds to the curvature bound for gravitational catalysis.

For $\xi_{k_{\mathrm{IR}}}=0$, the zero of the curve marks the curvature bound, since positive values of $\mathcal{A}$ are compatible with the absence of chiral symmetry breaking. From the numerical analysis we obtain the curvature bound,

$$
\left.\frac{\kappa}{k_{\mathrm{IR}}}\right|_{p=2} \leq 1.8998,\left.\quad \frac{\kappa}{k_{\mathrm{IR}}}\right|_{p \rightarrow \infty} \leq 1.5757
$$

for the two limiting regulators, showing that the full solutions deviate from the approximated ones by about $40 \%$.

A finite $\xi_{k_{\mathrm{IR}}}$ parameter corresponds to a linear vertical shift of the graph in Fig. 1 and a corresponding shift of the zero crossing marking the curvature bound. Figure 2 shows the curvature of the effective potential at the origin

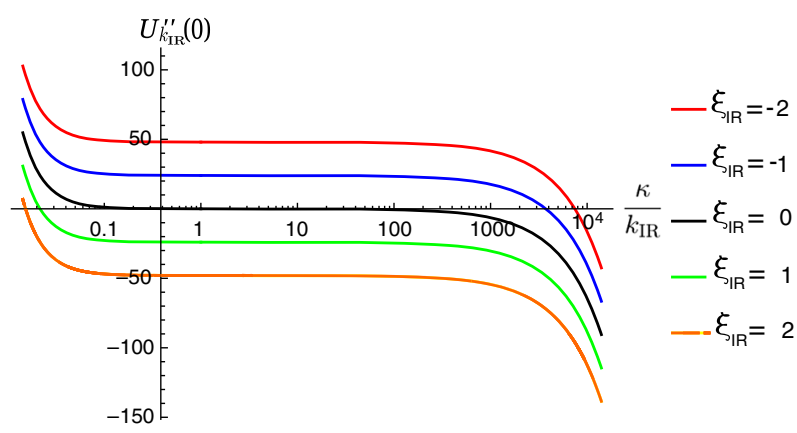

FIG. 2. Scaling behavior of the curvature of the effective potential at the origin (normalized by $N_{\mathrm{f}} \kappa^{2} / 2$ ) as a function of the curvature parameter $\kappa / k_{\mathrm{IR}}$ for the case $p=2$ and different values of the marginal coupling $\xi_{k_{\mathrm{IR}}}$. Positive values are compatible with the existence of light chiral fermions. The zero crossing marks the curvature bound which is strengthened for increasing values of $\xi_{k_{\mathrm{IR}}}$. 
(normalized by $N_{\mathrm{f}} \kappa^{2} / 2$ ) as a function of the curvature parameter $\kappa / k_{\mathrm{IR}}$ for various values of $\xi_{k_{\mathrm{IR}}}$. Positive values are compatible with the existence of light chiral fermions.

\section{HIGHER DIMENSIONS}

$$
\text { A. } D=6
$$

It is instructive to also study curvature bounds in higher dimensions. Perturbative nonrenormalizability implies that further relevant operators and thus physical couplings have to be accounted for; still, for any finite dimension, also the number of additional couplings is finite at mean-field level. As before, we have to pay attention only to those operators that couple to the masslike term in the effective potential. Other operators do not directly take influence on the curvature bound for chiral symmetry.

In $D=6$ dimensional spacetime, one further divergence of this type is encountered requiring to consider one more physical parameter. As before, the divergences are in correspondence with the small-proper-time expansion of the heat kernel for which we need to retain only the 0 -th order of the hyperbolic cotangent expansion inside the heat kernel,

$$
\begin{aligned}
K_{T}^{\mathrm{div}} & =\frac{1}{(4 \pi T)^{3}} \int_{0}^{\infty} d u e^{-u^{2}} u\left(u^{2}+\kappa^{2} T\right)\left(u^{2}+4 \kappa^{2} T\right) \\
& =\frac{1}{(4 \pi T)^{3}}\left(1+\frac{5}{2} \kappa^{2} T+2 \kappa^{4} T^{2}\right) .
\end{aligned}
$$

The divergencies associated with the curvature-dependent terms are controlled by initial conditions for the two operators

$$
\left.U_{\Lambda}\right|_{\phi^{2} R, \phi^{2} R^{2}}=N_{\mathrm{f}} \xi_{\Lambda} \phi_{0}^{2} R+N_{\mathrm{f}} \chi_{\Lambda} \phi_{0}^{2} R^{2} .
$$

Adding these two operators to the terms arising from Eq. (37), yields the following contributions to the masslike term in the effective potential:

$$
\begin{aligned}
\left.U_{k_{\mathrm{R}}}^{\mathrm{div}}\right|_{0} ^{2}= & -\frac{N_{\mathrm{f}} \phi_{0}^{2}}{2}\left\{\frac{\Lambda^{4}-k_{\mathrm{IR}}^{4}}{2(4 \pi)^{3}} \Gamma\left(1-\frac{2}{p}\right)\right. \\
& +\kappa^{2}\left[60 \xi_{\Lambda}+5 \frac{\Lambda^{2}-k_{\mathrm{IR}}^{2}}{2(4 \pi)^{3}} \Gamma\left(1-\frac{1}{p}\right)\right] \\
& \left.+\kappa^{4}\left[-1800 \chi_{\Lambda}+\frac{4}{(4 \pi)^{3}} \log \left(\frac{\Lambda}{k_{\mathrm{IR}}}\right)\right]\right\} \\
\equiv & -\frac{N_{\mathrm{f}} \phi_{0}^{2}}{2}\left\{\frac{1}{\bar{\lambda}_{\mathrm{cr}}}-\frac{k_{\mathrm{IR}}^{4}}{(4 \pi)^{3}} \Gamma\left(1-\frac{2}{p}\right)\right. \\
& \left.-2 \xi_{k_{\mathrm{IR}}} R-2 \chi_{k_{\mathrm{IR}}} R^{2}\right\} .
\end{aligned}
$$

Here, we have used that $\kappa^{2}=\frac{|R|}{D(D-1)}=\frac{|R|}{30}$ in $D=6$, and identified the critical coupling

$$
\bar{\lambda}_{\mathrm{cr}}=\frac{2(4 \pi)^{3}}{\Lambda^{4} \Gamma\left(1-\frac{2}{p}\right)} .
$$

The parameter $\xi_{\Lambda}$ has positive mass dimensions $\left(\left[\xi_{\Lambda}\right]=2\right)$ and thus the operator $\phi_{0}^{2} R$ is now a power-counting relevant operator, while $\phi_{0}^{2} R^{2}$ is marginal and the corresponding coupling $\chi_{\Lambda}$ has vanishing mass dimensions. The curvature-dependent terms in the last line of Eq. (39) are finite and need to be fixed by a measurement. As before, the divergence hidden in the critical coupling will be balanced by the initial condition for the bare coupling $\bar{\lambda}_{\Lambda}$.

This concludes the analytical treatment of the divergent parts. The remaining regular part of the effective potential can then be integrated straightforwardly by numerical means as in the $D=4$ case. In order to stay away from regulator artifacts, we choose the regulator parameter in the range $p \in[4, \infty]$. With the usual assumption of subcriticality, the dependence of the resulting masslike term of the effective potential (normalized by $N_{\mathrm{f}} \kappa^{2} / 2$ ) as a function of the curvature parameter $\kappa / k_{\mathrm{IR}}$ for the case $p=4$ and all further couplings $\xi_{k_{\mathrm{IR}}}, \chi_{k_{\mathrm{IR}}}$ set to zero is depicted in Fig. 3.

For a fair comparison of the curvature bounds for different spacetime dimensions, two conditions need to be met: (1) the physical parameters have to be chosen such that the relevant operator content is comparable, (2) the same $p$ parameter needs to be employed for the regularization procedure.

For the first condition, we simply set all independent scalar-curvature couplings to zero, $\xi_{k_{\mathrm{IR}}}=0=\chi_{k_{\mathrm{IR}}}$. For the second condition, we first check $p=4$ for numerical simplicity. This results in

$$
\begin{aligned}
& \frac{\kappa}{k_{\mathrm{IR}}} \leq 1.7039, \quad D=4, \quad \xi_{k_{\mathrm{IR}}}=0, \\
& \frac{\kappa}{k_{\mathrm{IR}}} \leq 1.2763, \quad D=6, \quad \xi_{k_{\mathrm{IR}}}=0, \quad \chi_{k_{\mathrm{IR}}}=0 .
\end{aligned}
$$

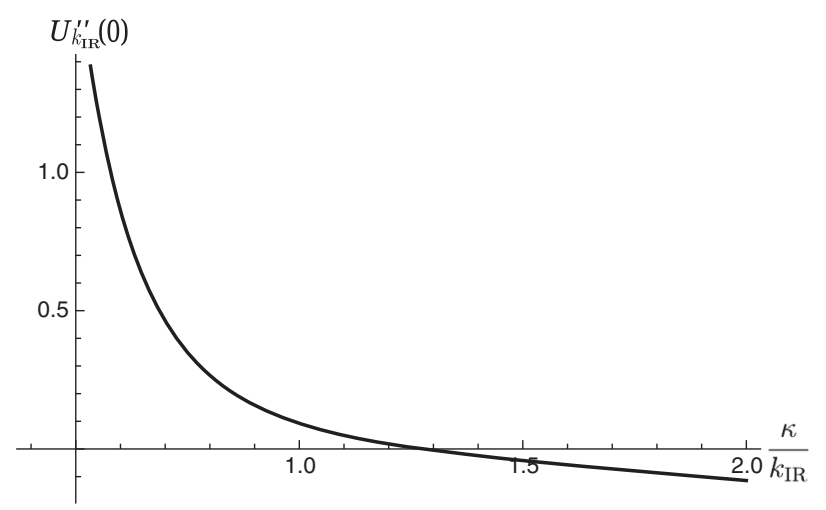

FIG. 3. $D=6$ scaling behavior of the curvature of the effective potential at the origin (normalized by $N_{\mathrm{f}} \kappa^{2} / 2$ ) as a function of the curvature parameter $\kappa / k_{\mathrm{IR}}$ for the case $p=4$ and $\xi_{k_{\mathrm{IR}}}=0=\chi_{k_{\mathrm{IR}}}$. Positive values are compatible with the existence of light chiral fermions. The zero crossing marks the curvature bound. 
The same analysis can be performed in the $p \rightarrow \infty$ limit. This scenario can be implemented noticing that the cutoff function reduces to a Heaviside $\theta$ function centered in $T=\frac{1}{k^{2}}$ and its derivative is therefore a Dirac $\delta$ distribution. In six dimensions, we obtain

$$
\left.\frac{\kappa}{k_{\mathrm{IR}}}\right|_{p \rightarrow \infty} \leq 1.0561
$$

We observe that, for both values of $p$, the bound for the dimensionless curvature parameter decreases with increasing the spacetime dimensions [compare with Eq. (36)]. We verify this circumstantial evidence in the next section for all odd dimensions. A general discussion follows below.

\section{B. Odd dimensions: $D=\mathbf{2 n}+1$}

The odd dimensional case is more easily analytically accessible thanks to the absence of the hyperbolic cotangent in the heat kernel [cf. (A11) and (A12)]. In line with the preceding studies, we associate the curvature bound with a possible sign change of the masslike term in the effective potential. Thus, it suffices to focus on the $\phi_{0}^{2}$ order of the effective potential. Inserting (A11) into (12) and expanding in powers of the field, we obtain

$$
\begin{aligned}
\left.U_{k_{\mathrm{IR}}}\right|_{\phi_{0}^{2}}= & \left.U_{\Lambda}\right|_{\phi_{0}^{2}}-\frac{N_{\mathrm{f}} \phi_{0}^{2}}{2}\left[\frac{4 p \kappa^{D-2}}{(4 \pi)^{\frac{D}{2}} \Gamma\left(\frac{D}{2}\right)}\right. \\
& \times \int_{\frac{k_{\mathrm{R}}}{\kappa}}^{\frac{\Lambda}{\kappa}} d \sigma \int_{0}^{\infty} d t t^{p-\frac{D}{2}} \sigma^{2 p-1} e^{-\left(\sigma^{2} t\right)^{p}} \\
& \left.\times \int_{0}^{\infty} d u e^{-u^{2}} \prod_{j=\frac{1}{2}}^{\frac{D}{2}-1}\left(u^{2}+j^{2} t\right)\right]
\end{aligned}
$$

where we have defined the dimensionless integration variable as $\sigma=\frac{k}{\kappa}$ and $t=\kappa^{2} T$. The effective potential can be decomposed into the following building blocks:

$U_{k_{\mathrm{IR}}}=\left.U_{\Lambda}\right|_{\kappa=0}+\left.U_{k_{\mathrm{IR}}}\right|_{\kappa=0}+U_{k_{\mathrm{IR}}, \kappa}^{\mathrm{reg}}+\left.U_{\Lambda}\right|_{\phi^{2} R^{n}}+\left.U_{k_{\mathrm{IR}}}\right|_{\phi^{2} R^{n}}$,

which we discuss separately in the following, concentrating on the masslike term $\sim \phi_{0}^{2}$. The first two terms correspond to the contribution which is present in flat space. By renormalizing the fermionic self-interaction, these terms exhibit the balance between the bare coupling $\bar{\lambda}_{\Lambda}$ and the leading cutoff divergence. The latter arises from the monomial containing the highest power of $u$ in the product in Eq. (43) $\sim u^{D-1}$, and can be summarized in the definition of the critical coupling

$$
\bar{\lambda}_{\mathrm{cr}}=\frac{(4 \pi)^{\frac{D}{2}}(D-2)}{2 \Lambda^{D-2} \Gamma\left(1-\frac{D}{2 p}+\frac{1}{p}\right)} .
$$

For the flat-space part, we thus obtain

$$
\begin{aligned}
& \left.U_{\Lambda}\right|_{\phi_{0}^{2}, \kappa=0}+\left.U_{k_{\mathrm{IR}}}\right|_{\phi_{0}^{2}, \kappa=0} \\
& \quad=\frac{N_{\mathrm{f}} \phi_{0}^{2}}{2}\left[\frac{1}{\bar{\lambda}_{\Lambda}}-\frac{1}{\bar{\lambda}_{\mathrm{cr}}}+\frac{2 \Gamma\left(1-\frac{D}{2 p}+\frac{1}{p}\right)}{(4 \pi)^{\frac{D}{2}}(D-2)} k_{\mathrm{IR}}^{D-2}\right] .
\end{aligned}
$$

The only a priori UV-regular term in Eq. (43) comes from the $u$-independent monomial arising from the product inside the last integral. It contains the relevant curvature dependence for gravitational catalysis:

$$
\begin{aligned}
\left.U_{k_{\mathrm{IR}}, \kappa}^{\mathrm{reg}}\right|_{\phi_{0}^{2}}= & -\frac{N_{\mathrm{f}} \phi_{0}^{2}}{2} \frac{4 p \kappa^{D-2}}{(4 \pi)^{\frac{D}{2}} \Gamma\left(\frac{D}{2}\right)} \\
& \times \int_{\frac{\mathrm{k}_{\mathrm{R}}}{\kappa}}^{\frac{\Lambda}{\kappa}} d \sigma \int_{0}^{\infty} d t t^{p-\frac{D}{2}} \sigma^{2 p-1} e^{-\left(\sigma^{2} t\right)^{p}} \\
& \times \int_{0}^{\infty} d u e^{-u^{2}} \frac{\Gamma^{2}\left(\frac{D}{2}\right)}{\pi} t^{\frac{D-1}{2}} \\
= & -\frac{N_{\mathrm{f}} \phi_{0}^{2}}{2} \frac{2 \Gamma\left(\frac{D}{2}\right) \Gamma\left(1+\frac{1}{2 p}\right)}{(4 \pi)^{\frac{D}{2}} \sqrt{\pi}} \frac{\kappa^{D-1}}{k_{\mathrm{IR}}}
\end{aligned}
$$

where we have taken the limit $\Lambda \rightarrow \infty$ in the last line.

All other monomials in the product of Eq. (43) carry UV divergencies, thus indicating the necessity to provide initial conditions for further operators. In total, we need $\frac{D-3}{2}$ operators with scalar-curvature couplings and correspondingly many physical parameters to be fixed by a measurement. The required operators are of the form $N_{\mathrm{f}} \xi_{\Lambda, m} \phi^{2} R^{n}$. Here, we choose conventions such that the index $m$ corresponds to a specific monomial in the above expression and $\xi_{\Lambda, m}$ parametrizing the initial condition for the bare coupling to be fixed. In order to analyze these contributions, we represent the polynomial part of the heat kernel as

$$
\prod_{j=\frac{1}{2}}^{\frac{D}{2}-1}\left(u^{2}+j^{2} t\right)=\sum_{m=0}^{\frac{D-1}{2}} C_{m} u^{2 m} t^{\frac{D-1}{2}-m},
$$

where $C_{m}$ denotes the numerical coefficients arising from the product. The resulting curvature dependence for each $m$ then results in a power $R^{n}$ with $n=\frac{D-1}{2}-m$. The $m=0$ term corresponds to the regular monomial computed in (47), while the $m=\frac{D-1}{2}$ term equals the curvatureindependent part of the heat kernel, already dealt with in Eq. (46). The remaining terms with $1 \leq m \leq(D-3) / 2$ in combination with the additional bare scalar-curvature operators thus make up for the last two terms in our decomposition (44) of the effective potential, yielding 


$$
\begin{aligned}
\left.U_{\Lambda}\right|_{\phi_{0}^{2} R^{n}}+\left.U_{k_{\mathrm{IR}}}\right|_{\phi_{0}^{2} R^{n}}= & -N_{\mathrm{f}} \phi_{0}^{2} \sum_{m=1}^{\frac{D-3}{2}} \kappa^{D-1-2 m} \\
& \times\left\{(-1)^{\frac{D-1}{2}-m-1} \xi_{\Lambda, m}[D(D-1)]^{\frac{D-1}{2}-m}\right. \\
& +\frac{C_{m} \Gamma\left(m+\frac{1}{2}\right) \Gamma\left(1-\frac{m}{p}+\frac{1}{2 p}\right)}{(4 \pi)^{\frac{D}{2}} \Gamma\left(\frac{D}{2}\right)(2 m-1)} \\
& \left.\times\left(\Lambda^{2 m-1}-k_{\mathrm{IR}}^{2 m-1}\right)\right\} \\
& \equiv-N_{\mathrm{f}} \phi_{0}^{2} \sum_{m=1}^{\frac{D-3}{2}}(-1)^{\frac{D-1}{2}-m-1} \kappa^{D-1-2 m} \xi_{k_{\mathrm{IR}}, m} .
\end{aligned}
$$

As before, the $\Lambda$-dependent terms combine with the bare couplings such that the long-range couplings $\xi_{k_{\mathrm{IR}}, m}$ are formed; for a physical system, the latter are finite and have to be fixed by a measurement. It is clear that possible curvature bounds will depend on these couplings. For the reason of comparing theories with different dimensionality, we set all these couplings to zero $\xi_{k_{\mathrm{R}}, m}=0$ at the scale $k_{\mathrm{IR}}$. Let us study two cases explicitly.

\section{1. $D=5$}

Inserting Eqs. (46) and (47) into Eq. (44) and using that Eq. (49) gives a vanishing contribution for $\xi_{k_{\mathrm{I}}, m}=0$, it is straightforward to obtain the following result for the masslike term of the scale-dependent effective potential in $D=5$ dimensional spacetime:

$$
\begin{aligned}
\left.U_{k_{\mathrm{IR}}}^{\mathrm{D}=5}\right|_{\phi_{0}^{2}}= & \frac{N_{\mathrm{f}} \phi_{0}^{2}}{2}\left[\frac{1}{\bar{\lambda}_{\Lambda}}-\frac{1}{\bar{\lambda}_{\mathrm{cr}}}+\frac{\Gamma\left(1-\frac{3}{2 p}\right)}{48 \pi^{\frac{5}{2}}} k_{\mathrm{IR}}^{3}\right. \\
& \left.-\frac{3 \Gamma\left(1+\frac{1}{2 p}\right)}{64 \pi^{\frac{5}{2}}} \frac{\kappa^{4}}{k_{\mathrm{IR}}}\right] .
\end{aligned}
$$

Assuming again a subcritical coupling as initial condition of the flow, we can identify the bound for the ratio between the curvature parameter and the averaging scale, below which symmetry breaking is not catalyzed gravitationally:

$$
\left(\frac{\kappa}{k_{\mathrm{IR}}}\right)^{4} \leq \frac{4}{9} \frac{\Gamma\left(1-\frac{3}{2 p}\right)}{\Gamma\left(1+\frac{1}{2 p}\right)} .
$$

In order to stay away from artifacts arising from insufficient regulators, we choose $p$ in the interval $p \in[2, \infty]$. For the two extremal cases, we have

$$
\begin{array}{ll}
\frac{\kappa}{k_{\mathrm{IR}}} \leq \frac{2}{\sqrt{3}} \simeq 1.154 & \text { for } p=2, \\
\frac{\kappa}{k_{\mathrm{IR}}} \leq \sqrt{\frac{2}{3}} \simeq 0.816 & \text { for } p=\infty .
\end{array}
$$

\section{2. $D=7$}

Similarly, the masslike term of the effective potential in $D=7$ dimensional spacetime reads

$$
\begin{aligned}
\left.U_{k_{\mathrm{IR}}}^{\mathrm{D}=7}\right|_{\phi_{0}^{2}}= & \frac{N_{\mathrm{f}} \phi_{0}^{2}}{2}\left[\frac{1}{\bar{\lambda}_{\Lambda}}-\frac{1}{\bar{\lambda}_{\mathrm{cr}}}+\frac{\Gamma\left(1-\frac{5}{2 p}\right)}{320 \pi^{\frac{7}{2}}} k_{\mathrm{IR}}^{5}\right. \\
& \left.-\frac{15 \Gamma\left(1+\frac{1}{2 p}\right)}{512 \pi^{\frac{7}{2}}} \frac{\kappa^{6}}{k_{\mathrm{IR}}}\right] .
\end{aligned}
$$

This time, a range of admissible regulators includes $p \in[3, \infty]$. Assuming a subcritical coupling, we can again read off the curvature bounds which for the extremal regulators are given by

$$
\begin{aligned}
& \frac{\kappa}{k_{\mathrm{IR}}} \leq 0.928, \quad \text { for } p=3, \\
& \frac{\kappa}{k_{\mathrm{IR}}} \leq 0.689, \quad \text { for } p=\infty .
\end{aligned}
$$

\section{Dimensional dependence}

As is obvious from all these examples, the curvature bound arises from a competition between the screening of the long-range modes parametrized by the last term in Eq. (46) and the dominant curvature term given by Eq. (47). For general $D$, we need to use the regulator with $p \rightarrow \infty$ to ensure that we stay away from regularization artifacts in any $D$. In order to perform a meaningful comparison, we set all possible nonzero scalar-curvature interactions terms $\sim \xi_{k_{\mathrm{IR}}, m}$ to zero. For this, the curvature bound can be expressed as follows:

$$
\frac{\kappa}{k_{\mathrm{IR}}} \leq \frac{1}{\sigma_{0}} \equiv\left(\frac{\sqrt{\pi}}{\Gamma\left(\frac{D}{2}\right)(D-2)}\right)^{\frac{1}{D-1}}
$$

exhibiting a monotonically decreasing behavior as is visible in Fig. 4. Asymptotically, the bound decays as $\sim 1 / \sqrt{D}$.

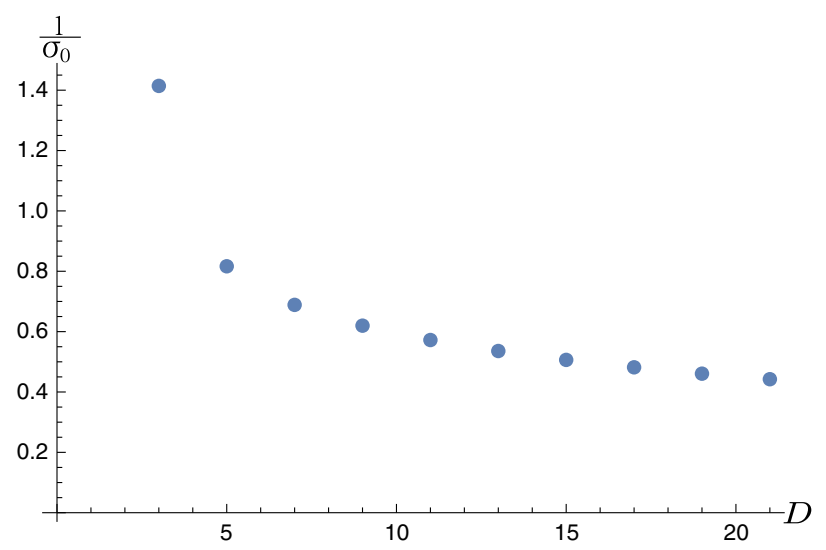

FIG. 4. Curvature bound (56) as a function of the spacetime dimensions in the odd-dimensional case for the regulator with $p \rightarrow \infty$. 
Of course, in the presence of further nonzero scalarcurvature interaction terms $\sim \xi_{k_{\mathrm{IR}}, m}$, the bound can be shifted in both directions depending on the precise parameter values.

\section{APPLICATION: ASYMPTOTICALLY SAFE GRAVITY}

As an illustration for the application of our curvature bound, we use a specific quantum gravity scenario in $D=4$ dimensional spacetime: asymptotically safe gravity [35-41,82]. In this scenario, Einstein's gravity arises as the low-energy limit of a quantum field theory of the metric, the high-energy behavior of which is controlled by a non-Gaussian fixed point in the space of relevant couplings. For simplicity, we confine ourselves to the theory space spanned by the Einstein-Hilbert action. A more comprehensive analysis suggests the existence of one further relevant operator with an overlap with an $R^{2}$-term in the action [83-96]. For a first glance at the consequences of the curvature bound, we also ignore the influence of the scalarcurvature operator $\sim \phi^{2} R$, which is, in principle, calculable within asymptotic safety from the fermionic operator content, i.e., schematically $\sim(\bar{\psi} \psi)^{2} R$.

In the modern functional renormalization group approach [97] to asymptotically safe gravity [37], one studies a scale-dependent effective action $\Gamma_{k}$ governing the dynamics of the expectation values of the field degrees of freedom, typically using a background-field gauge with a fiducial but arbitrary background metric. In the simple Einstein-Hilbert truncation, the background metric itself is a solution to the equation of motion derived from the scale-dependent effective action [70],

$$
R_{\mu \nu}\left(\langle g\rangle_{k}\right)=\bar{\Lambda}_{k}\left\langle g_{\mu \nu}\right\rangle_{k},
$$

where $\bar{\Lambda}_{k}$ denotes the scale-dependent cosmological constant, and $k$ is the coarse-graining or resolution scale, at which the spacetime is considered. Here, we have assumed the absence of any explicit matter sources. The asymptotic safety scenario provides us with a prediction for the RG trajectories for the cosmological constant $\bar{\Lambda}_{k}$, as well as for the UV fixed-point value $\lim _{k \rightarrow \infty} \bar{\Lambda}_{k} / k^{2}=\lambda_{*}$ being a finite number. In the fixed-point regime, the solution to Eq. (57) is given by

$$
\frac{R}{k^{2}}=4 \lambda_{*}
$$

This shows that the sign of the curvature in the fixedpoint regime is dictated by the sign of the fixed-point value of the cosmological constant. Equation (58) exemplifies the self-similarity property of physical observables in the fixed-point regime: the curvature is proportional to the scale $k$ at which the curvature is measured. While the fixed-point value $\lambda_{*}$ comes out positive in pure-gravity computations, it can change sign for an increasing number of fermionic degrees of freedom. Hence, the spacetime structure appears locally as negatively curved for large $N_{\mathrm{f}}$. The asymptotic safety scenario including matter degrees of freedom hence predicts that a local patch of spacetime in the (trans-Planckian) fixed-point regime satisfies

$$
\frac{\kappa^{2}}{k^{2}}=\frac{\left|\lambda_{*}\right|}{3}, \quad \text { for } \lambda_{*}<0 .
$$

Now, the precise value of the fixed point $\lambda_{*}$ is scheme dependent, see, e.g., [98-103] for comparative studies. With regard to Eq. (58) this is natural, since the result of a curvature measurement is expected to depend on the coarse-graining procedure that is used to average over metric fluctuations. This is precisely the type of scheme dependence, we expect to cancel the scheme dependence of our curvature bounds in order to arrive at a schemeindependent answer to the question as to whether or not there is gravitational catalysis in a given theory.

For the remainder of the section, we simply identify the gravitational RG coarse-graining scale $k$ with the scale $k_{\mathrm{IR}}$ used for our curvature bounds and use results obtained in the asymptotic-safety literature. In fact, the typical fixedpoint scenario can already be discovered within a simple one-loop calculation [41,99], yielding the fixed-point values for the cosmological constant and the dimensionless Newton constant

$$
\lambda_{*}=\frac{3}{4} \frac{2+d_{\lambda}}{46-d_{g}}, \quad g_{*}=\frac{12 \pi}{46-d_{g}} .
$$

Here, we used the results obtained from a so-called type IIa cutoff [41]. The two parameters $d_{g}$ and $d_{\lambda}$ are determined by the number of (free) matter degrees of freedom,

$d_{g}=N_{\mathrm{S}}-4 N_{\mathrm{V}}+2 N_{\mathrm{f}}, \quad d_{\lambda}=N_{\mathrm{S}}+2 N_{\mathrm{V}}-4 N_{\mathrm{f}}$,

where $N_{\mathrm{S}}$ denotes the number of real scalar fields, $N_{\mathrm{V}}$ the number of gauge vector bosons and-as before- $-N_{\mathrm{f}}$ the number of Dirac fermion flavors.

For gravitational catalysis to be potentially active at all, we need a negative fixed-point value $\lambda_{*}<0$, implying

$$
N_{\mathrm{f}}>\frac{1}{2}+\frac{N_{\mathrm{S}}}{4}+\frac{N_{\mathrm{V}}}{2} .
$$

This criterion is satisfied for the standard model with $N_{\mathrm{S}}=4, N_{\mathrm{V}}=12$ and $N_{\mathrm{f}}=45 / 2$, as well as typical generalizations with right-handed neutrino components, axion or simple scalar dark matter models. It is also generically satisfied for supersymmetric models; for instance, for the minimal supersymmetric standard model (MSSM) with two Higgs doublets, we have $N_{\mathrm{S}}=53$, $N_{\mathrm{V}}=12$ and $N_{\mathrm{f}}=65 / 2$. This exemplifies that the curvature bound should be monitored in asymptotically safe gravity-matter systems. However, the criterion (62) is 
typically not satisfied for GUT-like nonsupersymmetric theories where the contribution from larger number of gauge bosons and Higgs fields for the necessary symmetry breaking exceeds that of the fermion flavors.

For a given number of scalars and vectors, increasing the number of flavors drives the fixed point $\lambda_{*}$ towards more negative values. Using Eq. (59) with $k=k_{\mathrm{IR}}$, the averaged curvature can eventually violate the curvature bound. Hence, the curvature bound translates into an upper bound $N_{\mathrm{f}} \leq N_{\mathrm{f}, \mathrm{gc}}$ on the number of fermion flavors in order not to be inflicted by chiral symmetry breaking from gravitational catalysis. For instance, for a purely fermionic matter content, $N_{\mathrm{S}}=0=N_{\mathrm{V}}$, we find $N_{\mathrm{f}, \mathrm{gc}} \simeq 17.58$ for $p \rightarrow \infty$, and $N_{\mathrm{f}, \mathrm{gc}} \simeq 18.31$ for $p=2$, cf. Eq. (36). The scheme dependence of our curvature bound thus has only a mild influence on the critical fermion number.

Similarly, fixing the bosonic matter content to that of the standard model, $N_{\mathrm{S}}=4, N_{\mathrm{V}}=12$, the corresponding critical fermion number is $N_{\mathrm{f}, \mathrm{gc}} \simeq 35.97$ for $p \rightarrow \infty$. This would still allow for a fourth generation of standard model flavors, but exclude a fifth generation.

Interestingly, the MSSM with $N_{\mathrm{S}}=53$ and $N_{\mathrm{V}}=12$ would imply a critical flavor number of $N_{\mathrm{f}, \mathrm{gc}} \simeq 20.3$ far below the fermionic content of the model $N_{\mathrm{f}}=65 / 2$, thus indicating a possible tension between asymptotically safe gravity and a particle-physics matter content of that of the MSSM because of gravitational catalysis.

This analysis based on a simple one-loop calculation on the gravity side may be somewhat over-simplistic. In fact, a number of more sophisticated analyses have been performed for asymptotically safe gravity in conjunction with matter systems. A first study on the consistency of asymptotic safety with matter [46] was based on the background-field approximation with some improvements for the anomalous dimensions. Using their fixed-point results, we find $N_{\mathrm{f}, \mathrm{gc}} \simeq 8.21$ for a purely fermionic model $\left(N_{\mathrm{S}}=0=N_{\mathrm{V}}\right)$, and $N_{\mathrm{f}, \mathrm{gc}} \simeq 26.5$ for the standard model model with $N_{\mathrm{S}}=4, N_{\mathrm{V}}=12$ (and anomalous dimensions set to zero). The latter result includes the standard-model fermion content without and with right-handed neutrino partners, but does not offer room for a fourth generation. For the MSSM and other models there is not even a gravitational fixed point according to [46]. Even if we artificially reduce the number of fermion flavors, we do not find a suitable fixed point above $N_{\mathrm{f}} \simeq 17$. Here, $\lambda_{*}$ has become negative but the curvature bound is still satisfied.

The fixed-point scenario found in $[47,56]$ is different. The calculation distinguishes between the background field and the dynamical fluctuation field. The flow of the dynamical couplings which is driven by the dynamical correlators [104] is found to have a gravitational UV fixed point for any matter content that has been accessible in this study. This scenario hence does not rule out any particle-physics content from the side of UV compatibility with quantum gravity. Still, the predictions for the background-field couplings are qualitatively similar to those of [46]. The fixed-point results of [47] upon insertion into Eq. (59) and a comparison with the curvature bound suggest $N_{\mathrm{f}, \mathrm{gc}} \simeq 48.7$ for $p \rightarrow \infty$ for a purely fermionic model with $N_{\mathrm{S}}=0=N_{\mathrm{V}}$; for $p \rightarrow 2$, the results of [47] lead to $N_{\mathrm{f}, \mathrm{gc}} \simeq 50.9$.

Recently, an analysis of gravity-matter systems was performed in [51] using an ADM decomposition of the gravitational degrees of freedom, yielding an RG flow on foliated spacetimes. For both, gravitational as well as matter degrees of freedom, a type I regulator was used. As argued by the authors, the use of different regulators can be viewed as yielding a different map of the number of degrees of freedom $N_{\mathrm{S}}, N_{\mathrm{V}}$ and $N_{\mathrm{f}}$ onto the parameters $d_{g}$ and $d_{\lambda}$; e.g., for the type I regulator, one gets [41,51] $d_{g}=N_{\mathrm{S}}-N_{\mathrm{V}}-N_{\mathrm{f}}$. It has been argued that the type II regulator should be used for fermions in order to regulate the fluctuation spectrum of the Dirac operator in a proper fashion [41,105]. Hence, we use the flows of [51] but with a definition of the parameters $d_{g}$ and $d_{\lambda}$ as in Eq. (61). To leading order, this corresponds to a type I regularization of the gravity fluctuations but a type II regulator for the matter degrees of freedom.

In this case, the possible onset of gravitational catalysis for a purely fermionic model with $N_{S}=0=N_{V}$ occurs at a critical flavor number $N_{\mathrm{f}, \mathrm{gc}}=9.27$ for $p \rightarrow \infty\left(N_{\mathrm{f}, \mathrm{gc}}=\right.$ 9.84 for $p=2)$. For a standard-model like theory $\left(N_{S}=4\right.$, $\left.N_{V}=12\right)$, we have $N_{\mathrm{f}, \mathrm{gc}}=27.67 p \rightarrow \infty\left(N_{\mathrm{f}, \mathrm{gc}}=28.71\right.$ for $p=2$ ). Finally, the minimally supersymmetric extension of the standard model would lead to $N_{\mathrm{f}, \mathrm{gc}}=10.01$ for $p \rightarrow \infty\left(N_{\mathrm{f}, \mathrm{gc}}=10.27\right.$ for $\left.p=2\right)$, if we artificially allow $N_{\mathrm{f}}$ to vary independently in this model. Therefore the MSSM in this approximation is an example for a model where gravitational catalysis could lead to large-fermionmass generation in the trans-Planckian regime; in fact, if $N_{\mathrm{f}}$ is set to the physical value $N_{\mathrm{f}}=65 / 2$, the MSSM matter content in this setting does not lead to a fixed point suitable for asymptotically safe quantum gravity; see also [103].

We summarize the critical values for the fermion numbers $N_{\mathrm{f}, \mathrm{gc}}$ for $p \rightarrow \infty$ for the possible onset of gravitational catalysis derived within the various approximations for an asymptotically safe quantum gravity scenario in Table I. Whereas the standard model (e.g., also including righthanded neutrinos) satisfies the bound from gravitational catalysis in each of thee approximations, a standard model with a fourth fermion generation could already be affected by gravitational catalysis. Supersymmetric versions of the standard model show already some tension with the bound within asymptotically safe gravity.

Using the results of [51] as described above, we display the various regions in the space of matter theories parametrized by $d_{g}$ and $d_{\lambda}$; cf. Eq. (61), in Fig. 5 . In the upper orange-shaded region, the criterion analogous to (62) is not satisfied (in the calculation of [51], it corresponds to $d_{\lambda}>$ $-16 / 3)$; here, we expect a spacetime in the fixed-point 
TABLE I. Summary of the critical number of fermion species $N_{\text {f,gc }}$ below which particle theories are safe from chiral symmetry breaking through gravitational catalysis, using the $p \rightarrow \infty$ regulator. Results are shown for theories with a purely fermionic matter content (PF), the standard model and the MSSM artificially varying the number of fermions $\left(\mathrm{SM}+N_{\mathrm{f}}, \mathrm{MSSM}+N_{\mathrm{f}}\right)$. For an estimate of the UV properties of quantum spacetime, we use various literature results obtained within the asymptotic safety scenario of quantum gravity, see main text for details.

\begin{tabular}{lccc}
\hline \hline & \multicolumn{3}{c}{$N_{\mathrm{f}, \mathrm{gc}}$} \\
\cline { 2 - 4 } & $\mathrm{PF}$ & $\mathrm{SM}+N_{\mathrm{f}}$ & $\mathrm{MSSM}+N_{\mathrm{f}}$ \\
\hline $\begin{array}{c}\text { One-loop approx. } \\
\text { (type IIa) [41,99] }\end{array}$ & 17.58 & 35.97 & 20.3 \\
$\begin{array}{c}\text { Background-field } \\
\text { approximation [46] }\end{array}$ & 8.21 & 26.5 & no FP \\
$\begin{array}{c}\text { RG flow on foliated } \\
\text { spacetimes [51] }\end{array}$ & 9.27 & 27.67 & 10.01 \\
\begin{tabular}{c} 
Dynamical FRG [47] \\
\hline \hline
\end{tabular} & 48.7 & & \\
\hline
\end{tabular}

regime which is positively curved and thus not affected by gravitational catalysis. The curvature bound translates into a line in the $d_{g}, d_{\lambda}$ plane, with the (white) region above that line satisfying the bound. We observe that the lines for different regulators $p \in[2, \infty]$ are rather similar and deviate

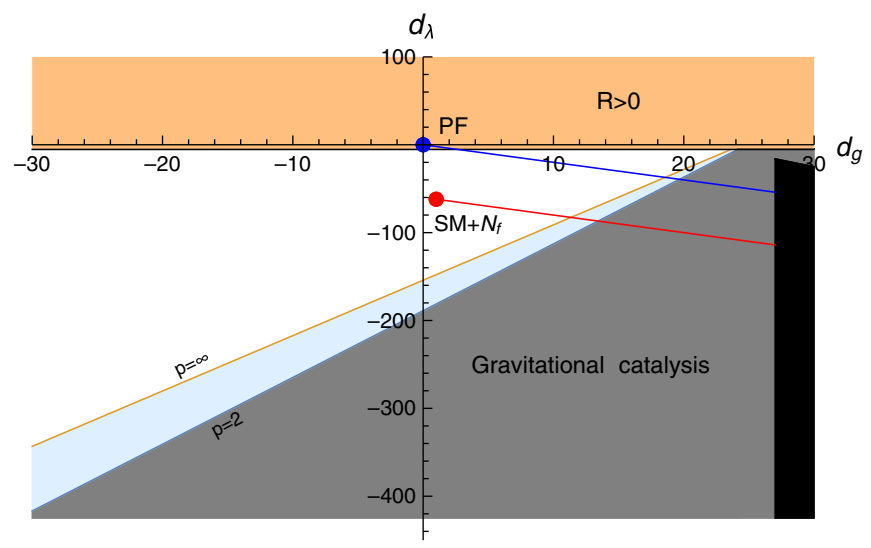

FIG. 5. Relevance of gravitational catalysis in different regions in the space of matter theories parametrized by $d_{g}$ and $d_{\lambda}$, cf. Eq. (61), using the results of [51] as an input from the asymptotic safety scenario for quantum gravity. In the bright orange region in the upper part, the criterion analogous to (62) is not satisfied, indicating that gravitational catalysis does not occur imposing no constraints. Our curvature bound results in a line with the (white) region above that line satisfying the bound; we observe a mild regulator dependence for different regulator parameters $p \in[2, \infty]$. Further lines show the location of purely fermionic particle models (PF) for an increasing number of flavors (blue), as well as the standard model (red dot) with additional flavor numbers $\left(\mathrm{SM}+N_{\mathrm{f}}\right)$. The gray region at the bottom indicates the region where chiral symmetry breaking and fermion mass generation because of gravitational catalysis can occur. The black region on the right does not have a non-Gaussian fixed point suitable for asymptotic safety [51]. significantly only for extreme particle numbers. The purely fermionic model (PF) and the standard model are represented by dots in the plane. The lines attached to the dots correspond to increasing the fermion number in these models. The purely fermionic model starts at $N_{\mathrm{f}}=0$, while the standard-model starts at its physical value $N_{\mathrm{f}}=45 / 2$. The MSSM with $N_{\mathrm{f}}=65 / 2$ would lie deep inside the black region to the right where no fixed point suitable for asymptotically safe gravity exists [51,103].

Let us close this section with two remarks: the first remark concerns the regularization scheme dependence which occurs at various places in this calculation. In case of a fully consistent calculation this scheme dependence would cancel in the final result for $N_{\mathrm{f}, \mathrm{gc}}$. However, since different parts in the present estimates are performed with different regulators, we observe various sources of scheme dependence. Whereas the scheme dependence arising from our mean-field RG calculation parametrized by $p$ is rather mild, a change of the regulator from type II to type I in the asymptotic safety scenario can change the dependence on the fermion flavor content significantly as studied in the literature [41,105]. Since our fermionic mean-field RG calculation corresponds to a type II regularization, we find it reassuring that a consistent use of type II regulators for the fermions leads to qualitatively and partly quantitatively similar results in the various approximations.

Second, the asymptotic safety scenario suggests that at least one further relevant operator of $R^{2}$ type should be included in the fixed-point regime. As this could take a significant quantitative influence on the effective equation of motion in the fixed-point regime, cf. Eq. (57), the relevance of the curvature bound for the asymptotic safety scenario may also change qualitatively. With these reservations in mind, the present discussion should be viewed as an example how the curvature bound from gravitational catalysis could potentially be used to constrain combined scenarios of quantum gravity and quantum matter.

\section{CONCLUSIONS}

We have studied gravitational catalysis of chiral symmetry breaking and fermion mass generation on patches of hyperbolic spaces, corresponding to negatively curved patches of AdS spacetimes in a Lorentzian setting. The general phenomenon of gravitationally catalyzed symmetry breaking has long been known to be driven by long-range modes and their sensitivity to the large-scale structure of negatively curved spacetimes. In this work, we have analyzed for the first time the competition between the screening of these modes by a gauge-invariant IR averaging scale $k_{\mathrm{IR}}$ and the effect of the presence of an averaged curvature on this scale. This competition leads to a bound on the local curvature parameter $\kappa \sim \sqrt{|R|}$ in units of the averaging scale $k_{\mathrm{IR}}$. Gravitational catalysis does not set in as long as the bound is satisfied. 
Built on RG-type arguments, our analysis applies to local patches of spacetime and hence does not require the whole spacetime to be hyperbolic, negatively curved or uniform. Rather the resulting bound applies to each patch of space or spacetime with an averaged negative curvature. Fermion modes in spacetime patches violating the bound can be subject to gravitational catalysis. The precise location of the onset of gravitational catalysis in parameter space depends also on further induced or fundamental interactions of the fermions. In case of chiral symmetry breaking through gravitational catalysis, the fermions generically acquire masses of the order of at least $k_{\mathrm{IR}}$ or larger depending on the relevance of further effective interactions.

An application of these findings to a possible highenergy regime of quantum gravity results in the following scenario: let us assume the existence of a, say Planck scale, regime where a metric/field theory description is already appropriate, but large curvature fluctuations are allowed to occur. Our bound disfavors the occurrence of patches of spacetime with large negative averaged curvature. In such patches, the generation of fermion masses of the order of $k_{\mathrm{IR}}$ could be triggered. Since $k_{\mathrm{IR}}$ itself can be of order Planck scale in such a regime, the fermion masses would generically be at the Planck scale upon onset of gravitational catalysis. Even worse, gravitational catalysis would naturally remove light fermions from the spectrum of particle physics models on such spacetimes. Therefore, we argue that our bounds apply to any quantum gravity scenario satisfying these assumptions that aims to be compatible with particle physics observations: if a quantum gravity scenario satisfies the bound, it is safe from gravitational catalysis in the matter sector; if not, the details of the fermion interactions matter. In the latter case, gravitational catalysis may still be avoided, if the interactions remain sufficiently weak.

As the curvature bounds refer to an IR cutoff scale $k_{\mathrm{IR}}$, they are naturally scheme dependent. In fact, this scheme dependence in the first place parametrizes the details of how the fermionic long-range modes are screened by the regularization scale. We observe that a finite curvature bound exists for any physically admissible regularization. Moreover, the shifts of the bound due to a change of the propertime regularization agrees with the behavior expected from the underlying propertime diffusion process. We therefore claim that the curvature bound has a scheme-independent meaning. A fully scheme-independent definition might eventually need to take the prescription for defining the averaged curvature of a local spacetime patch into account.

Having performed a mean-field-type RG analysis, our bounds may receive corrections from further fluctuations that may be relevant at the scale $k_{\mathrm{IR}}$ including further independent degrees of freedom or chiral-order-parameter fluctuations. Such corrections can go into both directions: finite magnetic fields or further interactions such as gauge or Yukawa forces typically enhance the approach to chiral symmetry breaking, whereas order-parameter fluctuations can have the opposite effect. Also, thermal fluctuations can inhibit the occurrence of a chiral condensate at sufficiently high temperature. Effects that trigger symmetry breaking can effectively be summarized in terms of finite bare fermionic self-interactions $\bar{\lambda}_{\Lambda}$ in our approach, whereas thermal fluctuations can be understood as moving the critical coupling to larger values [106,107].

We have been able to determine the curvature bound also in $D=3$ as well as in higher dimensions. In general odd dimensions, we have derived a simple closed form expression. Since different dimensions can exhibit a different number of relevant scalar-curvature operators and thus a different number of physical parameters, a meaningful comparison of theories in different dimensions is not straightforward. Assuming that all further physical parameters are essentially zero at the scale $k_{\mathrm{IR}}$, we observe that the resulting curvature bound decreases with $\sim 1 / \sqrt{D}$ for higher dimensions. This result inspires one to develop the following scenario: Let us assume that some fundamental theory of spacetime and matter can have a high-energy phase of arbitrary dimension and allows for a regime where a metric description applies. If the theory in addition exhibits fluctuating values of curvature $\kappa \sim \mathcal{O}(1)$ when averaged over local patches, our results suggest that it is unlikely to find higher-dimensional regions that admit massless or light fermions in the long-range physics. Upon the onset of gravitational catalysis, higher-dimensional regions would then generically go along with a massive fermionic particle content and without explicit chiral symmetry.

Unfortunately, results from quantum gravity scenarios that could be checked against our curvature bounds are rather sparse. Many approaches focus on the gravitational sector leaving matter, and fermions in particular, aside. One of the most developed approaches in this respect is asymptotically safe gravity. Concentrating on a simple picture for the UV regime of gravity using the EinsteinHilbert action as the scaling action, our curvature bound translates into a bound on the particle content of the matter sector. In particular, the number of fermion flavors becomes constrained in order to avoid gravitational catalysis. Our simple estimates based on various literature studies of asymptotically safe gravity with matter indicate that the standard model is compatible with asymptotically safe gravity and not affected by gravitational catalysis in the trans-Planckian regime. This statement is nontrivial insofar that the matter content together with the effective Einstein equation suggest negatively curved local patches of spacetime in the fixed-point regime. Still, the curvature is sufficiently weak to satisfy our curvature bound. As asymptotic freedom of the gauge interactions can be enhanced by gravitational fluctuations [53-56], also gauge-field induced catalysis is expected to play no dominant role in this scenario. By contrast, our estimates 
suggest that the standard model with an additional fourth flavor generation would not satisfy the curvature bound within asymptotic safety. In order to obtain more reliable estimates, the curvature dependence of correlation functions and its interdependence with the matter sector in the trans-Planckian fixed-point regime would be welcome.

This first application within a specific quantum gravity scenario demonstrates that our curvature bound may be usefully applicable also in the high-energy regime of other quantum gravity scenarios.

\section{ACKNOWLEDGMENTS}

We thank Astrid Eichhorn, Benjamin Knorr, Stefan Lippoldt, Jan Pawlowski, Frank Saueressig, Manuel Reichert, René Sondenheimer, and Omar Zanusso for valuable discussions. We are grateful to Astrid Eichhorn, Frank Saueressig, and Manuel Reichert for making their data and Mathematica files available to us. We acknowledge support by the DFG under Grants No. GRK1523/2 and No. Gi328/7-1.

\section{APPENDIX: HEAT KERNEL ON HYPERBOLIC SPACES}

For completeness, we summarize results for the heat kernel on hyperbolic spaces in this appendix, as they are needed for the present work. Following the derivation and conventions of $[80,81]$, we normalize the inverse radius $\kappa$ of the manifold to 1 , and reinstate this curvature parameter later. The heat kernel on a $D$-dimensional hyperbolic space can be written as

$$
\begin{gathered}
K\left(x, x^{\prime}, T\right)=\mathbb{U}\left(x, x^{\prime}\right) \hat{f}_{N}\left(d_{G}, T\right), \\
\hat{f}_{N}(y, T)=\frac{2^{D-3} \Gamma\left(\frac{D}{2}\right)}{\pi^{\frac{D}{2}+1}} \int_{0}^{\infty} \varphi_{\lambda}(y) e^{-T \lambda^{2}} \mu(\lambda) d \lambda,
\end{gathered}
$$

where $\mathbb{U}$ represents a parallel transport, and $\hat{f}_{N}$ is a scalar function satisfying the following equation:

$$
\begin{aligned}
0 & =\left(-\frac{\partial}{\partial T}+\square_{D}-\frac{R}{4}-\frac{D-1}{4} \tanh ^{2}(y)\right) \hat{f}_{N}(y) \\
& \equiv\left(-\frac{\partial}{\partial T}+L_{D}\right) \hat{f}_{N}(y),
\end{aligned}
$$

with $\square_{D}$ being the radial Laplacian. The eigenfunctions $\varphi_{\lambda}$ of the $L_{D}$ operator with eigenvalues $-\lambda^{2}$ can be written as

$L_{D} \varphi_{\lambda}=-\lambda^{2} \varphi_{\lambda}$,

$\varphi_{\lambda}(y)=\cosh \frac{y}{2}{ }_{2} F_{1}\left(\frac{D}{2}+i \lambda, \frac{D}{2}-i \lambda ; \frac{D}{2} ;-\sinh ^{2} \frac{y}{2}\right)$.

Here, ${ }_{2} F_{1}$ denotes the hypergeometric function, while the spectral measure $\mu(\lambda)$ reads

$$
\begin{aligned}
\mu(\lambda)= & \frac{\pi}{2^{2 D-4} \Gamma^{2}\left(\frac{D}{2}\right)} \\
& \times \begin{cases}\prod_{j=\frac{1}{2}}^{\frac{D}{2}-1}\left(\lambda^{2}+j^{2}\right), & D \text { odd } \\
\lambda \operatorname{coth}(\pi \lambda) \prod_{j=1}^{\frac{D}{2}-1}\left(\lambda^{2}+j^{2}\right), & D \text { even. }\end{cases}
\end{aligned}
$$

In the main text, cf. Sec. II, we only need the equal point limit of the heat kernel, with $x^{\prime} \rightarrow x$ and the geodesic distance $d_{G} \rightarrow 0$ goes to zero, i.e., $y \rightarrow 0$ in (A2). From Eq. (A5), it is clear that the coincident points limit leads to

$$
\lim _{y \rightarrow 0} \varphi_{\lambda}(y)=1,
$$

while the $\mathbb{U}$ reduces to the identity. Thus, we end up with

$$
K_{T}=\frac{2^{D-3} \Gamma\left(\frac{D}{2}\right)}{\pi^{\frac{D}{2}+1}} \int_{0}^{\infty} d \lambda e^{-T \lambda^{2}} \mu(\lambda) d \lambda .
$$

In order to reinstate the curvature parameter, we make contact with the flat space limit of the heat kernel, starting with the odd dimensional case. Plugging the definition of $\mu(\lambda)$ into Eq. (A8), we get upon substitution

$$
\begin{aligned}
K_{T}^{\text {odd }} & =\frac{2}{(4 \pi)^{\frac{D}{2}} \Gamma\left(\frac{D}{2}\right)} \int_{0}^{\infty} d \lambda e^{-T \lambda^{2}} \prod_{j=\frac{1}{2}}^{\frac{D}{2}-1}\left(\lambda^{2}+j^{2}\right) \\
& =\frac{2}{(4 \pi T)^{\frac{D}{2}} \Gamma\left(\frac{D}{2}\right)} \int_{0}^{\infty} d u e^{-u^{2}} \prod_{j=\frac{1}{2}}^{\frac{D}{2}-1}\left(u^{2}+j^{2} T\right),
\end{aligned}
$$

and similarly for an even dimensional background

$$
\begin{aligned}
K_{T}^{\text {even }}= & \frac{2}{(4 \pi T)^{\frac{D}{2}} \Gamma\left(\frac{D}{2}\right)} \int_{0}^{\infty} d u e^{-u^{2}} u \operatorname{coth}\left(\pi \frac{u}{\sqrt{T}}\right) \\
& \times \prod_{j=1}^{\frac{D}{2}-1}\left(u^{2}+j^{2} T\right) .
\end{aligned}
$$

Recalling that in flat spacetime the heat kernel in the coincident points limit reads $K_{T}=(4 \pi T)^{-1}$ with $T$ carrying mass dimension $[T]=-2$, we obtain the correct limit by rescaling the proper time inside the integrals by a sufficient power of the curvature parameter with $[\kappa]=1$; note that the integration variables has to remain dimensionless, $[u]=0$. We finally obtain

$$
\begin{aligned}
K_{T}^{\text {odd }}= & \frac{2}{(4 \pi T)^{\frac{D}{2}} \Gamma\left(\frac{D}{2}\right)} \int_{0}^{\infty} d u e^{-u^{2}} \prod_{j=\frac{1}{2}}^{\frac{D}{2}-1}\left(u^{2}+j^{2} \kappa^{2} T\right), \\
K_{T}^{\text {even }}= & \frac{2}{(4 \pi T)^{\frac{D}{2}} \Gamma\left(\frac{D}{2}\right)} \int_{0}^{\infty} d u e^{-u^{2}} u \operatorname{coth}\left(\pi \frac{u}{\kappa \sqrt{T}}\right) \\
& \times \prod_{j=1}^{\frac{D}{2}-1}\left(u^{2}+j^{2} \kappa^{2} T\right) .
\end{aligned}
$$


For an analytical approximation in even dimensions, the expansion of the integrand in the two limits $T \approx 0$ and $T \approx \infty$ are useful. For small $T$, we rewrite the hyperbolic cotangent as

$$
\operatorname{coth}\left(\pi \frac{u}{\kappa \sqrt{T}}\right)=1+2 \sum_{n=1}^{\infty} e^{-2 \frac{\pi n u}{\kappa \sqrt{T}}}
$$

The large $T$ regime corresponds to the small $u$ approximation of the hyperbolic cotangent, thus, it suffices to consider the first few terms in the Laurent expansion of $\operatorname{coth}\left(\pi \frac{u}{\kappa \sqrt{T}}\right)$ in order to capture the behavior of $K_{T}$ for $T$ around infinity,

$$
\operatorname{coth}\left(\pi \frac{u}{\kappa \sqrt{T}}\right)=\frac{\kappa \sqrt{T}}{\pi u}+\frac{\pi u}{3 \kappa \sqrt{T}}+\mathcal{O}\left(u^{3}\right)
$$

These two approximations are combined in Sec. III to identify an analytic approximation for the heat-kernel trace in four dimensions.
[1] I. L. Buchbinder and E. N. Kirillova, Sov. Phys. J. 32, 446 (1989).

[2] I. L. Buchbinder and E. N. Kirillova, Int. J. Mod. Phys. A 04, 143 (1989).

[3] T. Inagaki, T. Muta, and S. D. Odintsov, Mod. Phys. Lett. A 08, 2117 (1993).

[4] I. Sachs and A. Wipf, Phys. Lett. B 326, 105 (1994).

[5] E. Elizalde, S. Leseduarte, and S. D. Odintsov, Phys. Rev. D 49, 5551 (1994).

[6] E. Elizalde, S. Leseduarte, S. D. Odintsov, and Yu. I. Shilnov, Phys. Rev. D 53, 1917 (1996).

[7] S. Kanemura and H.-T. Sato, Mod. Phys. Lett. A 11, 785 (1996).

[8] T. Inagaki, Int. J. Mod. Phys. A 11, 4561 (1996).

[9] T. Inagaki and K.-i. Ishikawa, Phys. Rev. D 56, 5097 (1997).

[10] G. Miele and P. Vitale, Nucl. Phys. B494, 365 (1997).

[11] P. Vitale, Nucl. Phys. B551, 490 (1999).

[12] T. Inagaki, T. Muta, and S. D. Odintsov, Prog. Theor. Phys. Suppl. 127, 93 (1997).

[13] J. Hashida, S. Mukaigawa, T. Muta, K. Ohkura, and K. Yamamoto, Phys. Rev. D 61, 044015 (2000).

[14] E. V. Gorbar and V. P. Gusynin, Ann. Phys. (Amsterdam) 323, 2132 (2008).

[15] M. Hayashi, T. Inagaki, and H. Takata, arXiv:0812.0900.

[16] T. Inagaki and M. Hayashi, arXiv:1003.1173.

[17] E. V. Gorbar, Phys. Rev. D 61, 024013 (1999).

[18] E. V. Gorbar, Ukr. J. Phys. 54, 541 (2009).

[19] V. P. Gusynin, V. A. Miransky, and I. A. Shovkovy, Phys. Rev. D 52, 4718 (1995).

[20] V. P. Gusynin, V. A. Miransky, and I. A. Shovkovy, Phys. Lett. B 349, 477 (1995).

[21] V. P. Gusynin, V. A. Miransky, and I. A. Shovkovy, Nucl. Phys. B462, 249 (1996).

[22] S. P. Klevansky and R. H. Lemmer, Phys. Rev. D 39, 3478 (1989).

[23] K. G. Klimenko, Teor. Mat. Fiz. 89, 211 (1991) [Theor. Math. Phys. 89, 1161 (1991)].

[24] I. A. Shovkovy, Lect. Notes Phys. 871, 13 (2013).

[25] E. Elizalde, S. D. Odintsov, and A. Romeo, Phys. Rev. D 54, 4152 (1996).
[26] B. Geyer and S. D. Odintsov, Phys. Lett. B 376, 260 (1996).

[27] B. Geyer and S. D. Odintsov, Phys. Rev. D 53, 7321 (1996).

[28] V. C. Zhukovskii and I. V. Mamsurov, Theor. Math. Phys. 117, 1208 (1998).

[29] S. Sasagawa and H. Tanaka, Prog. Theor. Phys. 128, 925 (2012).

[30] D. Ebert, A. V. Tyukov, and V. C. Zhukovsky, Phys. Rev. D 80, 085019 (2009).

[31] D. D. Scherer and H. Gies, Phys. Rev. B 85, 195417 (2012).

[32] H. Gies and S. Lippoldt, Phys. Rev. D 87, 104026 (2013).

[33] A. Eichhorn and H. Gies, New J. Phys. 13, 125012 (2011).

[34] J. Meibohm and J. M. Pawlowski, Eur. Phys. J. C 76, 285 (2016).

[35] S. Weinberg, in Proceedings of the 1976 International School of Subnuclear Physics, Erice, Trapani, Sicily 1976 (Plenum Press, New York, 1978), p. 1.

[36] S. Weinberg, in General Relativity: An Einstein Centenary Survey, edited by S. W. Hawking and W. Israel (University Press, Cambridge, England, 1980), p. 790.

[37] M. Reuter, Phys. Rev. D 57, 971 (1998).

[38] M. Reuter and F. Saueressig, Phys. Rev. D 65, 065016 (2002).

[39] M. Niedermaier and M. Reuter, Living Rev. Relativity 9, 5 (2006).

[40] M. Reuter and F. Saueressig, New J. Phys. 14, 055022 (2012).

[41] R. Percacci, An Introduction to Covariant Quantum Gravity and Asymptotic Safety, http://www.worldscientific.com/ doi/pdf/10.1142/9789813207189.

[42] D. Dou and R. Percacci, Classical Quantum Gravity 15, 3449 (1998).

[43] R. Percacci and D. Perini, Phys. Rev. D 67, 081503 (2003).

[44] O. Zanusso, L. Zambelli, G. P. Vacca, and R. Percacci, Phys. Lett. B 689, 90 (2010).

[45] G. P. Vacca and O. Zanusso, Phys. Rev. Lett. 105, 231601 (2010). 
[46] P. Donà, A. Eichhorn, and R. Percacci, Phys. Rev. D 89, 084035 (2014).

[47] J. Meibohm, J. M. Pawlowski, and M. Reichert, Phys. Rev. D 93, 084035 (2016).

[48] K.-y. Oda and M. Yamada, Classical Quantum Gravity 33, 125011 (2016).

[49] A. Eichhorn, A. Held, and J. M. Pawlowski, Phys. Rev. D 94, 104027 (2016).

[50] Y. Hamada and M. Yamada, J. High Energy Phys. 08 (2017) 070.

[51] J. Biemans, A. Platania, and F. Saueressig, J. High Energy Phys. 05 (2017) 093.

[52] A. Eichhorn and A. Held, Phys. Rev. D 96, 086025 (2017).

[53] J.-E. Daum, U. Harst, and M. Reuter, J. High Energy Phys. 01 (2010) 084.

[54] S. Folkerts, D. F. Litim, and J. M. Pawlowski, Phys. Lett. B 709, 234 (2012).

[55] N. Christiansen and A. Eichhorn, Phys. Lett. B 770, 154 (2017).

[56] N. Christiansen, D. F. Litim, J. M. Pawlowski, and M. Reichert, arXiv:1710.04669.

[57] A. Eichhorn and S. Lippoldt, Phys. Lett. B 767, 142 (2017).

[58] M. Shaposhnikov and C. Wetterich, Phys. Lett. B 683, 196 (2010).

[59] U. Harst and M. Reuter, J. High Energy Phys. 05 (2011) 119.

[60] F. Bezrukov, M. Yu. Kalmykov, B. A. Kniehl, and M. Shaposhnikov, J. High Energy Phys. 10 (2012) 140.

[61] A. Eichhorn and A. Held, Phys. Lett. B 777, 217 (2018).

[62] A. Eichhorn and F. Versteegen, J. High Energy Phys. 01 (2018) 030.

[63] A. Eichhorn, arXiv:1709.03696.

[64] A. Eichhorn, A. Held, and C. Wetterich, arXiv:1711 .02949.

[65] C. Wetterich and M. Yamada, Phys. Lett. B 770, 268 (2017).

[66] B. Knorr and S. Lippoldt, Phys. Rev. D 96, 065020 (2017).

[67] N. Christiansen, K. Falls, J. M. Pawlowski, and M. Reichert, Phys. Rev. D 97, 046007 (2018).

[68] H. Gies, J. Jaeckel, and C. Wetterich, Phys. Rev. D 69, 105008 (2004).

[69] J. S. Schwinger, Phys. Rev. 82, 664 (1951).

[70] O. Lauscher and M. Reuter, J. High Energy Phys. 10 (2005) 050.

[71] R. P. Feynman, Phys. Rev. 80, 440 (1950).

[72] M. B. Halpern and W. Siegel, Phys. Rev. D 16, 2486 (1977).

[73] C. Schubert, Phys. Rep. 355, 73 (2001).

[74] H. Gies and K. Langfeld, Nucl. Phys. B613, 353 (2001).

[75] H. Gies and K. Langfeld, Int. J. Mod. Phys. A 17, 966 (2002).

[76] H. Gies, K. Langfeld, and L. Moyaerts, J. High Energy Phys. 06 (2003) 018.
[77] S.-B. Liao, Phys. Rev. D 53, 2020 (1996).

[78] S.-B. Liao, Phys. Rev. D 56, 5008 (1997).

[79] F. Gehring, H. Gies, and L. Janssen, Phys. Rev. D 92 , 085046 (2015).

[80] R. Camporesi, Commun. Math. Phys. 148, 283 (1992).

[81] R. Camporesi and A. Higuchi, J. Geom. Phys. 20, 1 (1996).

[82] D. F. Litim, Phys. Rev. Lett. 92, 201301 (2004).

[83] O. Lauscher and M. Reuter, Phys. Rev. D 66, 025026 (2002).

[84] A. Codello and R. Percacci, Phys. Rev. Lett. 97, 221301 (2006).

[85] P. F. Machado and F. Saueressig, Phys. Rev. D 77, 124045 (2008).

[86] D. Benedetti, P. F. Machado, and F. Saueressig, Mod. Phys. Lett. A 24, 2233 (2009).

[87] J. A. Dietz and T. R. Morris, J. High Energy Phys. 01 (2013) 108.

[88] K. Falls, D. F. Litim, K. Nikolakopoulos, and C. Rahmede, arXiv:1301.4191.

[89] K. Falls, D. F. Litim, K. Nikolakopoulos, and C. Rahmede, Phys. Rev. D 93, 104022 (2016).

[90] M. Demmel, F. Saueressig, and O. Zanusso, J. High Energy Phys. 08 (2015) 113.

[91] N. Ohta, R. Percacci, and G. P. Vacca, Phys. Rev. D 92 , 061501 (2015).

[92] K. Falls and N. Ohta, Phys. Rev. D 94, 084005 (2016).

[93] N. Christiansen, arXiv:1612.06223.

[94] T. Denz, J. M. Pawlowski, and M. Reichert, arXiv: 1612.07315.

[95] S. Gonzalez-Martin, T. R. Morris, and Z. H. Slade, Phys. Rev. D 95, 106010 (2017).

[96] K. G. Falls, C. S. King, D. F. Litim, K. Nikolakopoulos, and C. Rahmede, arXiv:1801.00162 [Phys. Rev. D (to be published)].

[97] C. Wetterich, Phys. Lett. B 301, 90 (1993).

[98] O. Lauscher and M. Reuter, Phys. Rev. D 65, 025013 (2001).

[99] A. Codello, R. Percacci, and C. Rahmede, Ann. Phys. (Amsterdam) 324, 414 (2009).

[100] H. Gies, B. Knorr, and S. Lippoldt, Phys. Rev. D 92 , 084020 (2015).

[101] N. Ohta, R. Percacci, and A. D. Pereira, J. High Energy Phys. 06 (2016) 115.

[102] K. Falls, Phys. Rev. D 96, 126016 (2017).

[103] N. Alkofer and F. Saueressig, arXiv:1802.00498.

[104] N. Christiansen, B. Knorr, J. Meibohm, J. M. Pawlowski, and M. Reichert, Phys. Rev. D 92, 121501 (2015).

[105] P. Donà and R. Percacci, Phys. Rev. D 87, 045002 (2013).

[106] J. Braun and H. Gies, J. High Energy Phys. 06 (2006) 024.

[107] J. Braun, J. Phys. G 39, 033001 (2012). 